\title{
Three and higher dimensional autonomous formations: Rigidity, persistence and structural persistence ${ }^{\text {ts }}$
}

\author{
Changbin $\mathrm{Yu}^{\mathrm{a}, \mathrm{b}, *}$, Julien M. Hendrickx ${ }^{\mathrm{c}}$, Barış Fidan ${ }^{\mathrm{a}, \mathrm{b}}$, \\ Brian D.O. Anderson ${ }^{\mathrm{a}, \mathrm{b}}$, Vincent D. Blondel ${ }^{\mathrm{c}}$ \\ ${ }^{a}$ National ICT Australia Ltd., Locked Bag 8001, Canberra ACT 2601, Australia \\ ${ }^{\mathrm{b}}$ Research School of Information Sciences and Engineering, The Australian National University, Canberra ACT 2601, Australia \\ ${ }^{\mathrm{c}}$ Department of Mathematical Engineering, Université Catholique de Louvain, Avenue Georges Lemaitre 4, B-1348 Louvain-la-Neuve, Belgium
}

Received 26 August 2005; received in revised form 17 January 2006; accepted 17 August 2006

Available online 10 January 2007

\begin{abstract}
In this paper, we generalize the notion of persistence, which has been originally introduced for two-dimensional formations, to $\mathfrak{R}^{d}$ for $d \geqslant 3$, seeking to provide a theoretical framework for real world applications, which often are in three-dimensional space as opposed to the plane. Persistence captures the desirable property that a formation moves as a cohesive whole when certain agents maintain their distances from certain other agents. We verify that many of the properties of rigid and/or persistent formations established in $\mathfrak{R}^{2}$ are also valid for higher dimensions. Analysing the closed subgraphs and directed paths in persistent graphs, we derive some further properties of persistent formations. We also provide an easily checkable necessary condition for persistence. We then turn our attention to consider some practical issues raised in multi-agent formation control in three-dimensional space. We display a new phenomenon, not present in $\mathfrak{R}^{2}$, whereby subsets of agents can behave in a problematic way. When this behaviour is precluded, we say that the graph depicting the multi-agent formation has structural persistence. In real deployment of controlled multi-agent systems, formations with underlying structurally persistent graphs are of interest. We analyse the characteristics of structurally persistent graphs and provide a streamlined test for structural persistence. We study the connections between the allocation of degrees of freedom (DOFs) across agents and the characteristics of persistence and/or structural persistence of a directed graph. We also show how to transfer DOFs among agents, when the formation changes with new agent(s) added, to preserve persistence and/or structural persistence.
\end{abstract}

(C) 2006 Elsevier Ltd. All rights reserved.

Keywords: Autonomous agents; Formations control; Structural persistence; Graph theory

\section{Introduction}

Multi-agent systems have attracted considerable attention recently as witnessed by the explosion of papers in the area (see e.g. Baillieul \& Suri, 2003; Das, Fierro, Kumar, \& Ostrowski, 2002; Eren, Anderson, Morse, Whiteley, \& Belhumeur, 2004;

\footnotetext{
Parts of this paper were presented at The First International Workshop on Multi-Agent Robotic Systems sponsored by the IFAC. This paper was recommended for publication in revised form by Associate Editor Ioannis Paschalidis under the direction of Editor Ian Petersen.

* Corresponding author. Tel.: +61 261256242; fax: +61 261258660 .

E-mail addresses: Brad.Yu@nicta.com.au (C. Yu), Hendrickx@inma.ucl.ac.be (J.M. Hendrickx), Baris.Fidan@nicta.com.au (B Fidan), Brian.Anderson@nicta.com.au (B.D.O. Anderson), Blondel@inma.ucl.ac.be (V.D. Blondel).
}

Gazi \& Passino, 2003; Jadbabaie, Lin, \& Morse, 2003; Lin, Francis, \& Maggiore, 2005; Marshall, Broucke, \& Francis, 1963; Olfati-Saber \& Murray, 2002, 2004; Ren \& Beard, 2004; Tanner, Pappas, \& Kumar, 2004). As illustrated in these references, many control tasks for multi-agent systems require coordinated motion of the agents in well-structured formations and hence acquisition and maintenance of the formation structure as well as the distance between nominated pairs of agents. The phenomena of formation acquisition and maintenance can be observed in nature in many forms, e.g., flocking birds, schooling fish, and swarming bees (see e.g. Hubbard, Babak, Sigurdsson, \& Magnusson, 2004; Janson, Middendorf, \& Beekman, 2005). Motivated by these observations, a significant number of recent studies have been performed in engineering applications including UAVs, roving robots, collection of ships, submarines, 
etc (see e.g. Ceccarelli, Di Marco, Garulli, \& Giannitrapani, 2005; Ihle, Jouffroy, \& Fossen, 2005).

Note here that we distinguish between two formation control tasks: (i) moving the whole formation from position A to $\mathrm{B}$; (ii) maintaining the shape of the formation while the whole formation is moving. These are clearly different problems, and this paper focuses on (ii). Note that in large biological formations in nature, it appears very unlikely that all members of the formation share a common view about where to go. Some members will just play the role of maintaining the shape, i.e., focus on (ii). This is consistent with a common idea in the literature concerning man-made formations, that some agents have leadership responsibility (and thus are concerned with moving the formation from A to B), and the rest (forming the majority) are followers.

Consequently, one needs to consider the following two questions in designing control structures and for such multi-agent systems: what can be measured, and what should be controlled?

Possible measurements include distance to neighbours, angular information between neighbours, angular information relative to fixed coordinates, e.g. vertical or north. For the control schemes, which are the subject of the paper, civil engineering structures give the first clue: if we maintain enough lengths/distances, we will retain rigidity, even with pinjoints. This indicates that one can imagine controlling lengths only, without worrying about active angle control in a formation. Note also that we do not need to control every single length; actively controlling some, the rest are consequentially maintained. By all means, the control effort (as measured by the number of actively controlled lengths) could be more than the minimum possible value for safety's sake, but in general the control effort should grow linearly with the number of formation elements. It is yet to be determined what is the best scheme, i.e., one based on lengths, or lengths and angles, etc. (and the issue of what is best will naturally depend on the sensor and possibly actuator settings). We know that if one can control based on lengths, one can then push the theory out to cope also with angular information of certain sorts (Eren, Whiteley, Morse, Belhumeur, \& Anderson, 2003).

The problem of maintaining the shape of a moving formation has been previously studied with graphs depicting the control architecture as follows (Eren et al., 2004; Olfati-Saber \& Murray, 2002): to each agent corresponds a vertex, and for each agent (vertex) pair $i, j$ there is an edge $(i, j)$ if the distance between $i$ and $j$ must be actively maintained. Note there is an implicit assumption that this is a joint task for both agents, which is often not the case for autonomous agents. The purpose of this paper is to focus on lengths/distances with the novel thrust being to hand the control task to just one agent rather than both, at the end of a particular length. In the recent control literature, the characterization of a system of the above type had started to be attempted under the name of rigidity of a directed graph (Baillieul \& Suri, 2003; Eren, Whiteley, Anderson, Morse, \& Belhumeur, 2005; Lin et al., 2005; Olfati-Saber \& Murray, 2002), and appears to have been first formalized using the notion of persistence of a directed graph (Hendrickx, Anderson, Delvenne, \& Blondel, 2005). This term is preferred because this notion is not equivalent to the simple application of the definition of rigidity to directed graphs.

In Section 2, the formal definition of persistence given in Hendrickx, Anderson et al. (2005) is generalized to $\mathfrak{R}^{d}$ for $d \geqslant 3$, seeking to provide a theoretical framework for real world applications, which often are in three-dimensional space as opposed to the plane. Persistence has the following intuitive meaning: a formation (and its underlying graph) is persistent if, provided that all the agents are trying to satisfy their distance constraints, they can in fact do that, and at the same time, a consequence of this fact is that the global structure of the formation is preserved, i.e., when the formation moves, it necessarily moves as a cohesive whole. We will see that rigidity of the underlying undirected graph is a necessary but not sufficient condition. This will lead us to the notion of constraint consistence of graph, which is the additional condition for a rigid graph (formation) to be persistent. Intuitively, a formation is constraint consistent if every agent is actually able to satisfy all its distance constraints provided that all the others are trying to do so. We will then argue that a graph is persistent if and only if it is rigid and constraint consistent. In Section 3, we generalize some of the main properties of persistent graphs to three- and higher dimensional graphs, drawing on established results in $\mathfrak{R}^{2}$ (Hendrickx, Anderson et al., 2005). We also define minimal persistence in $\mathfrak{R}^{d}$ for $d \geqslant 3$ analogously to minimal rigidity. We discuss some differences and similarities between the two notions, and give a characterization of minimally persistent graphs. In Section 4, we reason about the persistence of closed subgraphs of persistent graphs and use this reasoning to analyze the directed paths in persistent graphs (Hendrickx, Fidan, Yu, Anderson, \& Blondel, 2005). As results of this analysis, we present some further properties of persistent graphs and an easily checkable necessary condition for persistence.

We then use this material to focus on a practical issue related to persistence. We demonstrate that a persistent formation may suffer from a practical problem where any single agent can move to a position which satisfies the constraints on it once all the other agents are fixed but it is not possible to satisfy all the constraints on all the agents at the same time. In Section 5, we formally characterize this problem, which is closely associated with unsafe control of a formation in practical three-dimensional applications. We then introduce the notion of a structurally persistent graph, a class of persistent graphs free of the above problem. In real deployment of control of multi-agent systems, formations with underlying structurally persistent graphs are of interest. It is established in Section 5 importantly that in two dimensions, structural persistence and persistence are equivalent. We also provide a streamlined test of structural persistence.

In Section 6, we focus on the connections between allocation of degrees of freedom (DOFs) across agents and the characteristics of persistence and/or structural persistence of a directed graph. We also show how to transfer DOFs among agents, when the formation changes with new agent(s) added, to preserve persistence and/or structural persistence. We study cycle-free graphs in $\mathfrak{R}^{3}$ and show some more powerful results for this special case, including the existence of a linear time criterion to 
verify the cycle-free property and to decide persistence, which automatically assures structural persistence. The paper is ended with some concluding remarks given in Section 7.

\section{Rigidity and persistence}

In Hendrickx, Anderson et al. (2005), rigidity, persistence, and some other related notions have been defined for directed graphs in $\mathfrak{R}^{2}$. In this section, we generalize these definitions to be applicable for any space $\mathfrak{R}^{d}, d \in\{2,3, \ldots\}$. Some of the terms we use such as "rigidity" are undirected notions, i.e., notions that are defined for undirected graphs. Notions for undirected graphs can of course also be applied to directed graphs, e.g., we call a directed graph rigid if and only if its underlying undirected graph is rigid. Note that, in directed graphs, rigidity and the other undirected notions are not affected by modification of the edge directions.

In $\mathfrak{R}^{d}(d \in\{2,3, \ldots\})$, a representation of an undirected graph $G=(V, E)$ with vector set $V$ and edge set $E$ is a function $p: V \rightarrow \mathfrak{R}^{d}$. We say that $p(i) \in \mathfrak{R}^{d}$ is the position of the vertex $i$, and define the distance between two representations $p_{1}$ and $p_{2}$ of the same graph by

$d\left(p_{1}, p_{2}\right)=\max _{i \in V}\left\|p_{1}(i)-p_{2}(i)\right\|$.

A distance set $\bar{d}$ for $G$ is a set of distances $d_{i j}>0$, defined for all edges $(i, j) \in E$. A distance set is realizable if there exists a representation $p$ of the graph for which $\|p(i)-p(j)\|=d_{i j}$ for all $(i, j) \in E$. Such a representation is then called a realization. Note that each representation $p$ of a graph induces a realizable distance set (defined by $d_{i j}=\|p(i)-p(j)\|$ for all $(i, j) \in E$ ), of which it is a realization.

A representation $p$ is rigid if there exists $\varepsilon>0$ such that for all realizations $p^{\prime}$ of the distance set induced by $p$ and satisfying $d\left(p, p^{\prime}\right)<\varepsilon$, there holds $\left\|p^{\prime}(i)-p^{\prime}(j)\right\|=\| p(i)-$ $p(j) \|$ for all $i, j \in V$. (We say in this case that $p$ and $p^{\prime}$ are congruent). ${ }^{1} \quad$ A graph is said to be generically rigid if almost all its representations are rigid, in fact if there exists an open dense set in which all representations are rigid (Whiteley, 1997). Note that the reasons for which we only require almost all representations to be rigid instead of all of them will be detailed in Remark 4.

A widely used approach in the analysis of rigidity is the use of linear algebraic tools such as the rigidity matrix (Tay \& Whiteley, 1985; Whiteley, 1996a, 1997). For a graph $G=(V, E)$ in $\Re^{d}$, the rigidity matrix $R(G)$ of $G$ is defined as the $|E| \times d|V|$ matrix

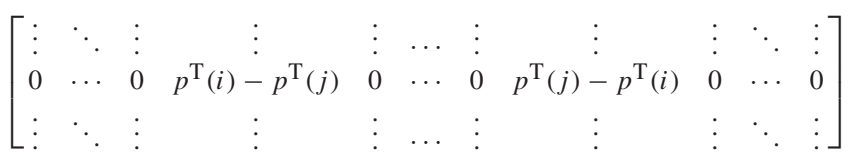

${ }^{1}$ The definition of rigidity, involving a quantifier $\varepsilon$, is standard (Tay \& Whiteley, 1985; Whiteley, 1996b). Any continuous large perturbation necessarily starts with a small perturbation. If small perturbations not preserving congruency exist, the same is true for large perturbations. Additionally, focusing on small perturbations allows study of the rigidity problem using linear algebraic methods, which is not the case for large perturbations. where each row

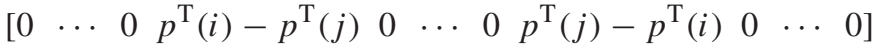

corresponds to an edge $(i, j) \in E, p^{\mathrm{T}}(i)-p^{\mathrm{T}}(j)$ is a row $d$ vector in the $d$ columns corresponding to vertex $i$. A standard result is that a graph $G$ with at least $d$ vertices is rigid if and only if for almost all representations, $R(G)$ has rank $d|V|-$ $d(d+1) / 2$, which is the maximum rank an $R(G)$ can have (Tay \& Whiteley, 1985; Whiteley, 1996a, 1997).

Another notion that is widely used in rigidity analysis is minimal rigidity. A graph is called minimally rigid if it is rigid and if there exists no rigid graph with the same number of vertices and a smaller number of edges. Equivalently, a graph is minimally rigid if it is rigid and if no single edge can be removed without losing rigidity. These two equivalent definitions of $\mathrm{min}$ imal rigidity lead to the following theorem, a version of which is presented as the "Necessary Counts Theorem" in Whiteley (1996a, 1997).

Theorem 1. If a graph $G=(V, E)$ in $\mathfrak{R}^{d}(d \in\{2,3, \ldots\})$ with at least $d$ vertices is rigid, then there exists a subset $E^{\prime}$ of edges such that $G^{\prime}=\left(V, E^{\prime}\right)$ is minimally rigid. This also implies the following:

- $\left|E^{\prime}\right|=d|V|-d(d+1) / 2$.

- Any subgraph $G^{\prime \prime}=\left(V^{\prime \prime}, E^{\prime \prime}\right)$ of $G^{\prime}$ with at least d vertices satisfies $\left|E^{\prime \prime}\right| \leqslant d\left|V^{\prime \prime}\right|-d(d+1) / 2$.

Proof. $G$ is rigid if and only if the rigidity matrix $R(G)$ has rank $d|V|-d(d+1) / 2$. Therefore, there exists a set of $d|V|-$ $d(d+1) / 2$ linearly independent rows in the matrix $R(G)$, corresponding to a set $E^{\prime} \subset E$ of $d|V|-d(d+1) / 2$ independent edges. Since the submatrix $R^{\prime}$ composed of these rows has rank $d|V|-d(d+1) / 2, G^{\prime}=\left(V, E^{\prime}\right)$ is rigid. Moreover, since $R^{\prime}$ is full rank, $G^{\prime}$ is minimally rigid and $\left|E^{\prime}\right|=d|V|-d(d+1) / 2$. Now, assume that there exists a subgraph $G^{\prime \prime}=\left(V^{\prime \prime}, E^{\prime \prime}\right)$ of $G^{\prime}$ for which $\left|E^{\prime \prime}\right|>d\left|V\left(E^{\prime \prime}\right)\right|-d(d+1) / 2$. Then the rows of $R^{\prime}$ corresponding to the edges in $E^{\prime \prime}$ have to be linearly dependent, noting that the entries of these rows corresponding to the vertices outside $V^{\prime \prime}$ are all zero. For otherwise, the matrix $R\left(G^{\prime \prime}\right)$ would have a rank greater than $d\left|V^{\prime \prime}\right|-d(d+1) / 2$, which as mentioned above is impossible for a rigidity matrix. However, this contradicts the fact that $R^{\prime}$ is full-rank. Therefore, each subgraph $G^{\prime \prime}=\left(V^{\prime \prime}, E^{\prime \prime}\right)$ of $G^{\prime}$ with at least $d$ vertices satisfies $\left|E^{\prime \prime}\right| \leqslant d\left|V^{\prime \prime}\right|-d(d+1) / 2$.

Lemma 2. Let $G=(V, E)$ be a minimally rigid graph in $\mathfrak{R}^{d}$ $(d \in\{2,3, \ldots\})$ and $G^{\prime}=\left(V^{\prime}, E^{\prime}\right)$ a subgraph of $G$ such that $\left|E^{\prime}\right|=d\left|V^{\prime}\right|-d(d+1) / 2$. Then, $G^{\prime}$ is minimally rigid.

Proof. Since $G$ is minimally rigid, the edge set $E$ is linearly independent. Therefore, $E^{\prime} \subseteq E$ is linearly independent as well. Since $\left|E^{\prime}\right|=d\left|V^{\prime}\right|-d(d+1) / 2$, this implies that the rigidity matrix of $G^{\prime}=\left(V^{\prime}, E^{\prime}\right)$ is full rank, i.e., $G^{\prime}$ is minimally rigid.

As mentioned above, rigidity is an undirected notion, and as noted in Hendrickx, Anderson et al. (2005), rigidity of a 
representation implies that if an external observer (or some physical properties) ensures that the distance between the positions of any pair of vertices connected by an edge remains constant, then all the sufficiently close realizations of the induced distance set are congruent to each other. But, in a typical system of autonomous agents, there is no such external observer. Each agent is only aware of its own distance constraints, and can "move freely" as long as these particular constraints are satisfied. The system therefore more naturally corresponds to a directed graph $G=(V, E)$, where each agent corresponds to a vertex in $V$, and for each agent (vertex) pair $i, j$ there is a directed edge $\overrightarrow{(i, j)} \in E$ from $i$ to $j$ if $i$ has a constraint on the distance it must actively maintain from $j$.

For example, agents that have only one constraint can move along a hyper-sphere centred on the position of the only other agent of which they are aware. So, it could happen that because one agent $i$ can lie anywhere on such a hyper-sphere, it becomes impossible for another agent $j$ to satisfy all its constraints, especially if $j$ has $d+1$ or more constraints. So, in order to have a more formal definition of persistence that guarantees the control of a system modelled using directed graph, we first need to characterize the fact that each agent is trying to keep the distances from its neighbours constant. Let us also fix desired distances $d_{i j}>0, \forall \overrightarrow{(i, j)} \in E$ and a representation $p$. We say that the edge $\overrightarrow{(i, j)} \in E$ is active if $\|p(i)-p(j)\|=d_{i j}$, i.e., the actual distance equals what is desired. We also say that the position of the vertex $i \in V$ is fitting for the distance set $\bar{d}$ if it is not possible to increase the set of active edges leaving $i$ by modifying the position of $i$ while keeping the positions of the other vertices unchanged. More formally, given a representation $p$, the position of vertex $i$ is fitting if there is no $p^{*} \in \mathfrak{R}^{d}$ for which the following strict inclusion holds:

$$
\begin{aligned}
& \left\{\overrightarrow{(i, j)} \in E:\|p(i)-p(j)\|=d_{i j}\right\} \\
& \quad \subset\left\{\overrightarrow{(i, j)} \in E:\left\|p^{*}-p(j)\right\|=d_{i j}\right\} .
\end{aligned}
$$

This condition intuitively means that the agent $i$ cannot satisfy additional distance constraints without breaking some that it already satisfies, as shown in the two-dimensional example in Fig. 1, which is drawn from Hendrickx, Anderson et al. (2005).

A representation of a graph is a fitting representation for a certain distance set $\bar{d}$ if all the vertices are at fitting positions for $\bar{d}$. Note that any realization is a fitting representation for its distance set. We can now give a formal definition of persistence:

A representation $p$ is persistent if there exists $\varepsilon>0$ such that every representation $p^{\prime}$ fitting for the distance set induced by $p$ and satisfying $d\left(p, p^{\prime}\right)<\varepsilon$ is congruent to $p$. A graph is then generically persistent if almost all its representations are persistent.

Next, we argue that a generically persistent graph in $\mathfrak{R}^{d}(d \in$ $\{2,3, \ldots\})$ is always generically rigid, and give a necessary and sufficient condition for a generically rigid graph to be generically persistent. This condition is called the generic constraint consistence of a graph. A representation $p$ is constraint consistent if there exists $\varepsilon>0$ such that any representation $p^{\prime}$ fitting for the distance set $\bar{d}$ induced by $p$ and satisfying $d\left(p, p^{\prime}\right)<\varepsilon$ is a realization of $\bar{d}$. Intuitively, the constraint consistence of a

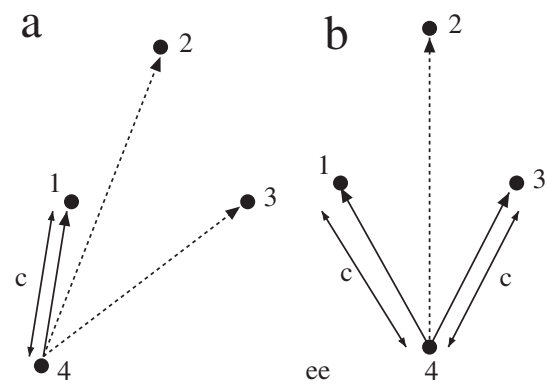

Fig. 1. Suppose that $d_{41}=q d_{42}=d_{43}=c$. The position of 4 in (a) is not fitting because it only makes $(\overrightarrow{4,1})$ active while there exists a position that would make both $(4,1)$ and $(4,3)$ active. On the other hand, its position in (b) is fitting because no point can be at the same time at a distance $c$ from 1,2 and 3 .

representation means that if each agent tries to satisfy its distance constraints (i.e., is at a fitting position), then all the distance constraints will be satisfied, or equivalently, no agent will be in a situation where it cannot satisfy some constraint. The illustration of such a situation in $\mathfrak{R}^{2}$ can be found in Hendrickx, Anderson et al. (2005). Again, we say that a graph is generically constraint consistent if almost all its representations are constraint consistent.

We have the following useful equivalences for directed graphs in any $d$-dimensional space $\Re^{d}(d \in\{2,3, \ldots\})$, which have already been established for $\mathfrak{R}^{2}$ in Hendrickx, Anderson et al. (2005).

Theorem 3. A representation in $\Re^{d}(d \in\{2,3, \ldots\})$ is persistent if and only if it is rigid and constraint consistent. A graph in $\mathfrak{R}^{d}(d \in\{2,3, \ldots\})$ is generically persistent if and only if it is generically rigid and generically constraint consistent.

The proof of Theorem 3 is identical to the two-dimensional case presented in Hendrickx, Anderson et al. (2005) and is omitted.

Remark 4. In our definitions of generic rigidity, persistence and constraint consistence, a graph has a generic property if almost all its representations have the property. Some pertinent discussion on our use of "generic" and "almost all" can be found in Hendrickx, Anderson et al. (2005) and Tay and Whiteley (1985). One reason for using these terms in $\mathfrak{R}^{d}(d \in$ $\{2,3, \ldots\})$ is to exclude the problems arising from having $d+1$ or more vertices lying on a $d_{1}$-dimensional hyper-surface where $d_{1} \leqslant d-1$. In the sequel however, we shall frequently assume the "generic" qualifier applies without explicit use of the word, when no misunderstanding is likely to occur.

\section{Characterization of persistent graphs}

In this section, we examine the properties of persistent graphs in three and higher dimensions. We present the fundamental results related to persistence and minimal persistence. These results are comparable to the properties of two-dimensional 
persistent graphs presented in Hendrickx, Anderson et al. (2005), and hence the corresponding propositions and lemmas are given as generic ones that are applicable to $\mathfrak{R}^{2}$ as well.

\subsection{Persistence}

We begin characterization of persistent graphs by giving a lower bound on the number of active edges, and an associated sufficient condition for a graph to be constraint consistent. In the sequel, $d_{G}^{-}(i)$ and $d_{G}^{+}(i)$ designate respectively the in- and out-degree of the vertex $i$ in the graph $G$. When no confusion is possible about the graph, we will use $d^{-}(i)$ and $d^{+}(i)$. Note also that the results given in this section and their proofs are very similar to the two-dimensional case presented in Hendrickx, Anderson et al. (2005).

Lemma 5. Let $p$ be a representation of a graph $G=(V, E)$ in $\mathfrak{R}^{d}(d \in\{2,3, \ldots\})$, and $i$ a vertex of this graph. If the position $p(i)$ does not lie on any $(d-1)$-dimensional hyper-plane containing $d$ or more of its neighbours, then there exists $\varepsilon>0$ such that in every representation $p^{\prime} \in B(p, \varepsilon) \triangleq\{\bar{p}: V \rightarrow$ $\left.\mathfrak{R}^{d} \mid d(p, \bar{p}) \leqslant \varepsilon\right\}$ fitting for the distance set induced by $p$, the number of active edges leaving $i$ is at least $\min \left(d, d^{+}(i)\right)$. Consequently, a graph in which all the vertices have an out-degree smaller than or equal to dis always generically constraint consistent.

Proof. We follow the same steps as in the proof of Lemma 1 in Hendrickx, Anderson et al. (2005). Let us consider a representation $p^{\prime}$ fitting for the distance set $\bar{d}$ induced by $p$. First, if the out-degree of the vertex $i$ is less than $d$ then the set of possible positions that could make all the edges leaving $i$ active is always non-empty, e.g., this set is equal to $\mathfrak{R}^{d}$ for $d^{+}(i)=0$, a circle for $d^{+}(i)=d-1$, etc. The position $p^{\prime}(i)$ will then be fitting if and only if all the $d^{+}(i)=\min \left(d, d^{+}(i)\right)$ edges are active.

Second, if the out-degree of $i$ is greater than or equal to $d$, we need the following result, which can be shown using simple geometric and continuity arguments:

Suppose there are given $d+1$ points $a_{1}, a_{2}, \ldots, a_{d+1} \in \mathfrak{R}^{d}$ that do not all lie on any $(d-1)$-dimensional hyper-plane. Let $d_{k l}$ denote the distance between each pair $a_{k}, a_{l}$ where $k \neq l$. There exists an $\varepsilon\left(a_{1}, a_{2}, \ldots, a_{d+1}\right)>0$ such that for any $a_{1}^{\prime}, a_{2}^{\prime}, \ldots, a_{d}^{\prime} \in \mathfrak{R}^{d}$ satisfying $\left\|a_{k}-a_{k}^{\prime}\right\|<\varepsilon\left(a_{1}, a_{2}, \ldots, a_{d+1}\right)$ for $k=1,2, \ldots, d$, there exists $a_{d+1}^{\prime} \in \mathfrak{R}^{d}$ such that $\| a_{k}^{\prime}-$ $a_{d+1}^{\prime} \|=d_{k(d+1)}$ for $k=1,2, \ldots, d$.

We can now show that

$$
\varepsilon_{i}=\min _{\left(i, j_{1}\right), \ldots,\left(i, j_{d}\right) \in E} \varepsilon\left(p(i), p\left(j_{1}\right), \ldots, p\left(j_{d}\right)\right)
$$

satisfies the required condition in the statement of Lemma 5. Let us indeed suppose that there is a representation $p^{\prime} \in$ $B(p, \varepsilon)$ such that less than $d$ active edges are leaving $i$, and take a set of $d$ edges $\overrightarrow{\left(i, j_{1}\right)}, \ldots, \overrightarrow{\left(i, j_{d}\right)}$, containing the active edges leaving $i$ if there are any. Observe that by hypothesis, $p(i), p\left(j_{1}\right), \ldots, p\left(j_{d}\right)$ do not all lie on any $(d-1)$-dimensional hyper-plane. By (2), there exists thus a point $p^{*}$ such that $\left\|p^{*}-p^{\prime}\left(j_{k}\right)\right\|=d_{i k}$ for $k=1, \ldots, d$, or equivalently a point $p^{*}$ such that the strict inclusion (1) holds. The position $p^{\prime}(i)$ and the representation $p^{\prime}$ are thus not fitting for $\bar{d}$, which contradicts our hypothesis.

Next, we show the second part of the result. Observe first that in almost all representations of the graph $G$, no vertex has a position lying on any $(d-1)$-dimensional hyper-plane containing $d$ or more of its neighbours. Let us consider such a representation $p$ of $G$ for which every vertex $i$ has an outdegree $d^{+}(i) \leqslant d$, and the induced distance set $\bar{d}$. If we take $\varepsilon^{\prime}<\varepsilon_{i}, \forall i \in V$ where the $\varepsilon_{i}$ comes from (2) for each vertex, then for any representation $p^{\prime} \in B\left(p, \varepsilon^{\prime}\right)$ fitting for $\bar{d}$, each vertex will be left by $\min \left(d, d^{+}(i)\right)=d^{+}(i)$ active edges, so that all the edges will be active. Every such $p^{\prime}$ is thus a realization of $\bar{d}$, and the representation $p$ is thus constraint consistent. As we already mentioned, this can be done for almost all representations of $G$, which is therefore also generically constraint consistent.

The next proposition which is the generalization of Propositions 1 and 2 in Hendrickx, Anderson et al. (2005) for any arbitrary dimension $d \in\{2,3, \ldots\}$, allows us to delete edges in a persistent (constraint consistent) graph and maintain persistence (constraint consistence).

Proposition 6. A persistent graph in $\mathfrak{R}^{d}(d \in\{2,3, \ldots\})$ remains persistent after deletion of any edge $\overrightarrow{(i, j)}$ for which $d^{+}(i) \geqslant d+1$. Similarly, a constraint consistent graph in $\mathfrak{R}^{d}$ $(d \in\{2,3, \ldots\})$ remains constraint consistent after deletion of any edge $\overrightarrow{(i, j)}$ for which $d^{+}(i) \geqslant d+1$.

Proof (Sketch). We follow the same steps as in the proof of the like Proposition 1 in Hendrickx, Anderson et al. (2005). Let $G=(V, E)$ be a persistent graph having a vertex $i$ with $d^{+}(i) \geqslant d+1$, and $G^{*}=\left(V, E^{*}\right)$ be the graph obtained by removing an edge $\overrightarrow{(i, j)}$ from $G$. Let us consider a realization $p$ of $G^{*}$ and the induced distance set $\bar{d}^{*}$. Observe that $p$ can also be viewed as a representation of $G$, and the induced distance set is then $\bar{d}=\bar{d}^{*} \cup\left\{d_{i j}\right\}$.We assume here that no vertex has a position that lies on a $(d-1)$-dimensional hyper-plane containing $d$ or more of its neighbours (and thus that Lemma 5 can be applied), which is the case for almost all realizations. We can first prove (A) that any fitting representation of $G^{*}$ for $\bar{d}^{*}$ sufficiently close to $p$ is also a fitting representation of $G$ for $\bar{d}$. This then allows us to prove in $(B)$ the persistence of $G^{*}$ in a relatively direct way.

An interesting corollary of Proposition 6 concerns the total number of DOFs, which we also call the total DOF count, in the graph. The number of degrees of freedom (DOF count) of a vertex is the maximal dimension, over all generic representations of the graph, of the set of possible fitting positions for this vertex. Effectively, the DOF count of the vertex $v$ is equal to $\max \left\{d-d^{+}(v), 0\right\}$. For example, in $\mathfrak{R}^{3}$, the DOF counts of the vertices with zero, one, and two out-degrees are respectively 3,2 , and 1 ; all the other vertices have zero DOF. Note that a vertex with zero DOF can have more than one possible fitting position. Observe indeed that, in almost all situations in 


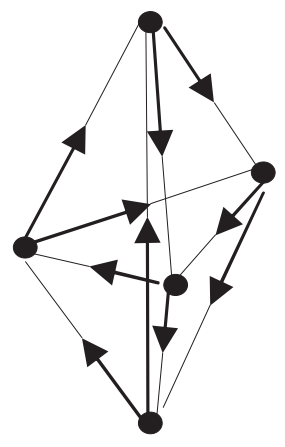

Fig. 2. A persistent graph in $\mathfrak{R}^{3}$ with all the vertices having out-degree 2 and hence 1-DOF.

$\mathfrak{R}^{d}$, there are two possible fitting positions for a vertex with out-degree $d$. However, since this set contains a finite number of points, its dimension is still 0 . The following result provides a natural bound on the total DOF count, i.e., the sum of the vertex DOF counts of a persistent graph.

Corollary 7. The total DOF count of a persistent graph in $\mathfrak{R}^{d}$ $(d \in\{2,3, \ldots\})$ can at most be $d(d+1) / 2$.

Proof. Observe first that removing an edge leaving a vertex with an out-degree larger than or equal to $d+1$ does not affect the number of degrees of freedom of this vertex. Let us now imagine that there exists a persistent graph $G=(V, E)$ for which this sum is larger than $d(d+1) / 2$. Using recursively Proposition 6 , we could obtain a persistent subgraph $G^{*}=\left(V, E^{*}\right)$ with the same total DOF count but without any vertex having an outdegree exceeding $d$. In $G^{*}$, the DOF count of a vertex $i$ is thus $d-d^{+}\left(i, G^{*}\right)$. So, if $F$ is the sum of the DOFs on all the vertices of the graph, we have $F=d|V|-\left|E^{*}\right| . F>d(d+1) / 2$ would then mean that $\left|E^{*}\right|<d|V|-d(d+1) / 2$, which by Theorem 1 is impossible for a persistent (and thus rigid) graph.

Remark 8. There are $d$-dimensional persistent graphs having a total DOF count less than $d(d+1) / 2$. Fig. 2 shows a threedimensional persistent graph each vertex of which has 1-DOF. The total DOF count for this example is 5, i.e., less than $3(3+$ 1) $/ 2$.

We have asserted in Proposition 6 that a persistent graph remains persistent after deletion of any edge $\overrightarrow{(i, j)}$ for which $d^{+}(i) \geqslant d+1$. Hence after successive deletions, we can thus reach in this way a persistent graph whose vertices all have an outgoing degree that is smaller than or equal to $d$. In the next theorem, which is analogous to Theorem 3 in Hendrickx, Anderson et al. (2005) stated for $\mathfrak{R}^{2}$, we assert that a graph is persistent if and only if all the graphs obtained in this way are rigid.

Theorem 9. A d-dimensional graph is persistent if and only if all those subgraphs, which are obtained by successively removing outgoing edges from vertices with out-degree larger thand until all such vertices have an out-degree equal to $d$, are rigid.
Proof (Sketch). We follow the similar steps as in the proof of Theorem 3 in Hendrickx, Anderson et al., 2005. Let us consider a $d$-dimensional graph $G=(V, E)$ and $\sum$ the set of all the subgraphs $S$ of $G$ satisfying for each vertex $i \in V$, $d_{S}^{+}(i)=\min \left(d_{G}^{+}(i), d\right)$. We can prove separately the following two implications:

- If $G$ is persistent, any $S \in \sum$ is rigid.

Since it is possible to obtain $S$ from $G$ only by removing edges leaving vertices with an out-degree larger or equal to $d+1$, Proposition 6 guarantees the persistence of $S$ and thus its rigidity.

- If every $S \in \Sigma$ is rigid, $G$ is persistent.

Using Theorem 1, having every $S \in \Sigma$ rigid and hence at least one rigid subgraph of $G$ implies that $G$ is rigid. Hence, we just need to, and indeed we can, prove the constraint consistence of $G$.

Theorem 9 provides a non-polynomial time algorithm to check the persistence of any $d$-dimensional graph for $d \in$ $\{2,3, \ldots\}$ : it is sufficient to check the rigidity of all subgraphs obtained by deleting the edges leaving vertices with out-degree larger than or equal to $d+1$ until all the vertices have an outdegree less or equal to $d$. An algorithm with a smaller complexity would be useful in the case of large graphs, especially if there is a high number of vertices with high out-degrees, but no such algorithm is currently available. More discussions on determining the persistence of two-dimensional directed graphs in polynomial time can be found in Hendrickx, Anderson et al. (2005). We also note the existence of a quadratic time algorithm for the cycle-free graphs in $\mathfrak{R}^{3}$ (Yu, Hendrickx, Fidan, \& Anderson, 2005), which can be generalized easily to any $d \in$ $\{2,3, \ldots\}$.

We also define the notion of minimal persistence, analogously to minimal rigidity defined in Section 2. A persistent graph in $\mathfrak{R}^{d}(d \in\{2,3, \ldots\})$ is said to be minimally persistent if no single edge can be removed without losing persistence. Obviously then, any persistent graph contains a minimally persistent subgraph with the same vertex set. However, an important difference between the concepts of minimal rigidity and minimal persistence is that a graph $G=(V, E)$ having a minimally persistent subgraph $G^{\prime}=\left(V, E^{\prime}\right)$ with the same vertex set $V$ is not necessarily persistent, as shown in the two-dimensional example in Fig. 3, which is drawn from Hendrickx, Anderson et al. (2005). More generally, unlike the case of rigidity, it is possible to obtain a non-persistent graph by adding edges (without adding any vertex) to a persistent graph. However, similar to minimal rigidity, we can show that the number of edges of a minimally persistent graph is uniquely determined by the number of its vertices. A necessary condition for a persistent graph to be minimally persistent, as Proposition 6 implies, is the absence of a vertex with an out-degree exceeding $d$. A sufficient condition, on the other hand, is minimal rigidity. Suppose indeed that one removes an edge from a persistent minimally rigid graph. The obtained graph would by definition not be rigid and hence not persistent. We can show that this condition is in fact also necessary. 


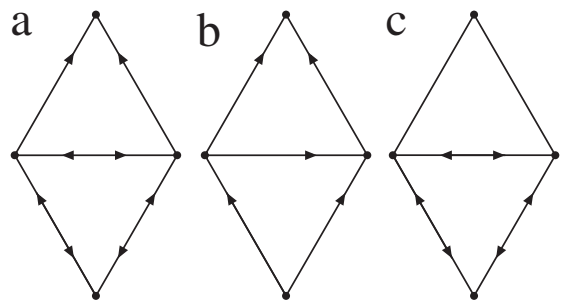

Fig. 3. The two-dimensional graph represented in (a) has a minimally persistent subgraph (b). However, it is not persistent because the subgraph represented in (c) and obtained via the deletion process of Theorem 9 is not rigid. In the corresponding multi-agent system, this could arise from a combination of unfortunate information architecture selections for the three agents of the cycle.

\section{Closed subgraphs and directed paths}

In this section, we focus on the directed paths in persistent graphs and analyse some related properties. As a part of this analysis, we reason about the persistence of closed subgraphs of minimally and non-minimally persistent graphs. An important outcome of our analysis will be an easily checkable necessary condition (Proposition 16) for persistence.

We begin with defining some notions that we use in the analysis frequently. Consider a directed graph $G=(V, E)$ in $\Re^{d}(d \in\{2,3, \ldots\})$ and a subgraph $G^{\prime}=\left(V^{\prime}, E^{\prime}\right)$ of $G . G^{\prime}$ is called a closed subgraph of $G$ if for any vertex $i \in V^{\prime}$, $\overrightarrow{(i, j)} \in E$ implies $j \in V^{\prime}$ and $\overrightarrow{(i, j)} \in E^{\prime}$. Equivalently, $G^{\prime}$ is a closed subgraph of $G$ if there is no directed path in $G$ starting from a vertex of $V^{\prime}$ and containing either a vertex or an edge that does not belong to $G^{\prime}$. The sum of DOF counts $\sum_{v \in V^{\prime}} \max \left\{d-d_{G}^{+}(v), 0\right\}$, where $d_{G}^{+}(v)$ denotes the out-degree of $v$ in the graph $G$, is called the total DOF count of $V^{\prime}$ with respect to $G$ and denoted by $S\left(V^{\prime}, G\right)$. Similarly, $d_{G^{\prime}}^{+}(v)$ (for any $\left.v \in V^{\prime}\right)$ and $S\left(V^{\prime}, G^{\prime}\right)$ denote, respectively, the out-degree of $v$ in $G^{\prime}$ and the total DOF count $\sum_{v \in V^{\prime}} \max \left\{d-d_{G^{\prime}}^{+}(v), 0\right\}$ of $V^{\prime}$ with respect to $G^{\prime}$. Note that if $V=V^{\prime}$, the definition is consistent with that given just prior to Corollary 7 for the total DOF count.

Remark 10. For a minimally persistent graph $G=(V, E)$, there holds $S(V, G)=d(d+1) / 2$.

Using these notions, we obtain the following propositions, which provide some criteria to check the existence of a directed path between given two vertices of a minimally or nonminimally persistent graph, one of which has a positive DOF count.

Lemma 11. Let $G=(V, E)$ be a minimally persistent graph in $\Re^{d}(d \in\{2,3, \ldots\})$. Any vertex $i$ that does not belong to a closed subgraph of $G$ having less than d vertices is connected by a directed path (leaving i) to any vertex $v$ of $G$ with positive DOF count, i.e., $d_{G}^{+}(v)<d$.

Proof. Suppose to obtain a contradiction that there exists a vertex pair $i, j \in V$ such that (i) $i$ does not belong to any closed subgraph of $G$ having less than $d$ vertices, (ii) $j$ has a positive DOF count, and (iii) there is no directed path from $i$ to $j$. Let $V^{\prime}$ denote the set of all the vertices $k \in V$ such that there is a directed path from $i$ to $k$, including the vertex $i$ itself, and $E^{\prime}$ the set of all the edges $e \in E$ which leave the vertices in $V^{\prime}$. Obviously, every edge in $E^{\prime}$ is incident to two vertices in $V^{\prime}$, i.e., $V\left(E^{\prime}\right)=V^{\prime} . G^{\prime}=\left(V^{\prime}, E^{\prime}\right)$ is thus a closed subgraph containing $i$, which by hypothesis implies that $\left|V^{\prime}\right| \geqslant d$. Moreover, since $j \notin V^{\prime}$, by definition of $V^{\prime}$ and by the hypothesis (ii), we have $S\left(V^{\prime}, G\right)<S(V, G)=d(d+1) / 2$ using Remark 10. Since $G^{\prime}$ is a closed subgraph of $G$, the DOF count of any $v \in V^{\prime}$ is the same for $G$ and $G^{\prime}$. Hence, $S\left(V^{\prime}, G^{\prime}\right)=S\left(V^{\prime}, G\right)<d(d+1) / 2$. Since every vertex has an out-degree less than or equal to $d$ in a minimally persistent graph, this leads to the inequality

$$
\begin{aligned}
\left|E^{\prime}\right|=\sum_{v \in V^{\prime}} d^{+}(v) & =\sum_{v \in V^{\prime}} d-S\left(V^{\prime}, G^{\prime}\right) \\
& >d\left|V^{\prime}\right|-d(d+1) / 2
\end{aligned}
$$

which contradicts Theorem 1, where the set $E^{\prime}$ above corresponds to $E^{\prime \prime}$ in Theorem 1 . Therefore, any vertex $i$ that does not belong to a closed subgraph of $G$ having less than $d$ vertices has to be connected by a directed path to all the vertices of $G$ with positive DOF count.

Remark 12. In $\Re^{d}(d \in\{2,3, \ldots\})$, if a vertex belongs to a closed subgraph containing less than $d$ vertices, its out-degree must be smaller than or equal to $d-2$.

Next, we extend the result given in Lemma 11 to arbitrary persistent graphs. In order to make the analysis easier, we introduce the notions of $k$-maximally connected closed subgraph and reachability subgraph. Given a directed graph $G=(V, E)$ in $\Re^{d}(d \in\{2,3, \ldots\})$ and an integer $k \leqslant|V|$, we call a connected closed subgraph $G_{c}$ of $G$ with less than or equal to $k$ vertices a k-maximally connected closed subgraph of $G$ if and only if $G_{c}$ is not a subgraph of any (other than $G_{c}$ itself) connected closed subgraph of $G$ with less than or equal to $k$ vertices. Consider a vertex $i \in V$ and the set $V^{\prime}$ of all the vertices that can be reached from $i$ (including the vertex $i$ ) by a directed path in $G$. Let $E^{\prime} \subseteq E$ the set of all the edges $e$ such that $e$ joins a pair of vertices in $V^{\prime}$. Then, the graph $G^{\prime}=\left(V^{\prime}, E^{\prime}\right)$ is called the reachability subgraph of $G$ for $i$. We note here that a similar reachability concept is introduced in Krumke and Noltemeier (2005).

Remark 13. Given a directed graph $G=(V, E)$ be in $\Re^{d}(d \in$ $\{2,3, \ldots\})$, for any vertex $i \in V$, the reachability subgraph of $G$ for $i$ is a closed subgraph of $G$. Moreover, it is easy to see that the reachability subgraph of $G$ for the vertex $i$ is the smallest closed subgraph of $G$ containing $i$, and equivalently, the intersection of all the closed subgraphs of $G$ containing $i$.

Theorem 14. Let $G=(V, E)$ be a persistent graph in $\Re^{d}(d \in$ $\{2,3, \ldots\})$ having at least $d$ vertices. If a vertex $v \in V$ belongs to a closed subgraph of $G$ containing less than d vertices, the out-degree of $v$ has to be smaller than or equal to $d-2$. For the set $V_{c}$ of all such vertices $v$, we have $\left|V_{c}\right| \leqslant d-1$. On the other 
hand, any vertex $v^{\prime} \in V$ that does not belong to any closed subgraph of $G$ having less than d vertices is connected by a directed path (leaving $v^{\prime}$ ) to all the vertices of $G$ with positive DOF count.

Proof. It is easy to see from the definition of closed subgraph that if $v \in V$ belongs to a closed subgraph containing less than $d$ vertices, then $d^{+}(v) \leqslant d-2$. In order to show that $\left|V_{c}\right| \leqslant d-1$, consider the set $\Gamma_{d}=\left\{\bar{G}_{i}=\left(\bar{V}_{i}, \bar{E}_{i}\right), i=1, \ldots, \bar{n}\right\}$ of all the $(d-1)$-maximally connected closed subgraphs of $G$. By applying the definition of a $k$-maximally connected closed subgraph, it is not hard to verify that $V_{c}=\bigcup_{i=1}^{\bar{n}} \bar{V}_{i}$ and hence $\left|V_{c}\right| \leqslant \bar{v} \stackrel{\Delta}{=} \sum_{i=1}^{\bar{n}}\left|\bar{V}_{i}\right|$. Let us consider an arbitrary $\bar{G}_{i}$. Since $\bar{G}_{i}$ is a closed subgraph of $G$, using the definition of total DOF count and Remark 12 we obtain

$S\left(\bar{V}_{i}, G\right)=S\left(\bar{V}_{i}, \bar{G}_{i}\right)=d\left|\bar{V}_{i}\right|-\left|\bar{E}_{i}\right|$.

Moreover, since the number of edges in $\bar{G}_{i}$ can at most be the one of the complete graph with $\left|\bar{V}_{i}\right|$ vertices, we have $\left|\bar{E}_{i}\right| \leqslant\left|\bar{V}_{i}\right|\left(\left|\bar{V}_{i}\right|-1\right) / 2$, which together with (4) implies that

$S\left(\bar{V}_{i}, G\right) \geqslant(d+1 / 2)\left|\bar{V}_{i}\right|-\left|\bar{V}_{i}\right|^{2} / 2$.

Taking the summation of (5) over $\Gamma_{d}$, we obtain

$\sum_{i=1}^{\bar{n}} S\left(\bar{V}_{i}, G\right) \geqslant(d+1 / 2) \bar{v}-\sum_{i=1}^{\bar{n}}\left|\bar{V}_{i}\right|^{2} / 2$.

Since $\left|\bar{V}_{i}\right| \leqslant d-1, \forall i$, we have

$\sum_{i=1}^{\bar{n}}\left|\bar{V}_{i}\right|^{2} \leqslant \max _{j}\left|\bar{V}_{j}\right| \sum_{i=1}^{\bar{n}}\left|\bar{V}_{i}\right| \leqslant(d-1) \bar{v}$

and hence

$\sum_{i=1}^{\bar{n}} S\left(\bar{V}_{i}, G\right) \geqslant(d+1 / 2) \bar{v}-(d-1) \bar{v} / 2$

$$
=(d / 2+1) \bar{v} .
$$

On the other hand, since there can be at most $d(d+1) / 2$ degrees of freedom in $\mathrm{G}$, we have

$\sum_{i=1}^{\bar{n}} S\left(\bar{V}_{i}, G\right) \leqslant d(d+1) / 2$.

(6) and (7) yield that $d(d+1) / 2 \geqslant(d / 2+1) \bar{v}$, i.e., $\bar{v} \leqslant d-$ $d /(d+2)$. Noting that $\bar{v}$ is integer, this implies $\left|V_{c}\right| \leqslant \bar{v} \leqslant d-1$.

Finally, to prove the last statement of the theorem, consider a vertex $k \in V$ with a positive DOF count in $G$. Since $G$ is persistent, by Proposition 6 and definition of minimal persistence, there exists a minimally persistent subgraph $G^{\prime}=\left(V, E^{\prime}\right)$ of $G$, having the same vertex set $V$ as $G$. Moreover, since $G^{\prime}$ is obtained by removing some edges from $G, k$ has a positive DOF count in $G^{\prime}$ as well. Suppose $v^{\prime}$ is as in the theorem statement. If $v^{\prime}$ does not belong to a closed subgraph of $G^{\prime}$ with less than $d$ vertices, then because of Lemma 11 there exists a directed path from $v^{\prime}$ to $k$ in $G^{\prime}$ and thus in $G$.
Let us now suppose that $v^{\prime}$ belongs to a closed subgraph of $G^{\prime}$ with less than $d$ vertices. By the hypothesis of the theorem, the reachability subgraph of $G$ for $v^{\prime}$ has to have at least $d$ vertices since it is a closed subgraph of $G$. In other words, at least $d$ vertices (including the vertex $v^{\prime}$ ) can be reached from $v^{\prime}$ by a directed path in $G$. Among these $d$ vertices, as we just observed in the first part of this proof, there is at least one vertex $\tilde{v}$ that does not belong to a closed subgraph of $G^{\prime}$ having less than $d$ vertices. The vertex $\tilde{v}$ is thus connected to $k$ by a directed path in $G^{\prime}$ and a fortiori in $G$, again because of Lemma 11, and hence there is a directed path from $v^{\prime}$ to $k$ in $G$ via $\tilde{v}$.

The following corollary, which immediately follows from Theorem 14, gives a more explicit criterion to check the existence of a directed path between given two vertices of a persistent graph in $\mathfrak{R}^{3}$, one of which has a positive DOF count.

Corollary 15. Let $G=(V, E)$ be a persistent graph in $\mathfrak{R}^{3}$ having at least three vertices. Any vertex $i$ is connected by a directed path (leaving $i$ ) to all the vertices of $G$ with positive DOF count unless one of the following holds:

(1) $i$ is a "leader", i.e., $d^{+}(i)=0$.

(2) $i$ is a "first follower", i.e., $d^{+}(i)=1$ and there exists a $j \in V$ such that $\overrightarrow{(i, j)} \in E$ and $d^{+}(j)=0$.

Using Theorem 14, we reach the following easily checkable necessary condition for persistence.

Proposition 16. Let $G=(V, E)$ be a persistent graph in $\Re^{d}$ $(d \in\{2,3, \ldots\})$ with at least $d$ vertices. Then all the closed subgraphs of $G$ having more than $d-1$ vertices are persistent (and hence every closed subgraph of $G$ for $d=2$ is persistent).

Proof. We consider two cases based on the out-degrees of the vertices in $V$. In Case 1 , we consider the persistent graphs $G=(V, E)$ such that no vertex $v \in V$ has an out-degree larger than $d^{2}$ and in Case 2, the proof of which builds on the proof of Case 1, we consider the persistent graphs with at least one vertex having out-degree larger than $d$.

- Case 1: $G=(V, E)$ is a persistent graph in $\mathfrak{R}^{d}$ with at least $d$ vertices such that no vertex $v \in V$ has an out-degree larger than $d$. Let $G^{\prime}=\left(V^{\prime}, E^{\prime}\right)$ be an arbitrary closed subgraph of $G$ containing more than $d-1$ vertices. The hypothesis of the proposition together with Lemma 5 imply that no vertex in $G^{\prime}$ has an out-degree larger than $d$ and thus that $G^{\prime}$ is constraint consistent. It remains then to prove the rigidity of $G^{\prime}$.

Since $G$ is rigid, due to Theorem 1, there exists an $E^{*} \subseteq E$ such that $G^{*}=\left(V, E^{*}\right)$ is minimally rigid, and hence (i) $\left|E^{*}\right|=d|V|-d(d+1) / 2$ and (ii) $\left|E^{* *}\right| \leqslant d \mid V\left(E^{* *} \mid-d(d+\right.$ 1)/2) for any $E^{* *} \subseteq E^{*}$. Let us consider $E^{\prime *}$ the restriction of $E^{*}$ to $G^{\prime}$, i.e., $E^{\prime *}=E^{*} \cap E^{\prime}$ and the graph $G^{* *}=\left(V^{\prime *}, E^{\prime *}\right)$

\footnotetext{
${ }^{2}$ There are persistent graphs in $\Re^{d}$ which are not minimally persistent but have all the vertices with out-degree less than or equal to $d$. Such a graph in $\mathfrak{R}^{3}$ is shown in Fig. 2 .
} 
where $V^{\prime *}=V\left(E^{\prime *}\right)$. We are going to prove that $G^{\prime *}$ is minimally rigid, which will imply the rigidity of $G^{\prime}$. We have $V^{\prime *}=V\left(E^{* *}\right) \subseteq V\left(E^{\prime}\right)=V^{\prime}$ and hence $\left|V^{\prime *}\right| \leqslant\left|V^{\prime}\right|$. Since $E^{\prime *} \subseteq E^{*}$ and $G^{*}$ is minimally rigid, Theorem 1 implies that $\left|E^{\prime *}\right| \leqslant d\left|V^{\prime *}\right|-d(d+1) / 2 \leqslant d\left|V^{\prime}\right|-d(d+1) / 2$.

If we prove that $\left|E^{\prime *}\right|$ cannot be less than $d\left|V^{\prime}\right|-d(d+$ 1) $/ 2$, we will have $\left|E^{* *}\right|=d\left|V^{* *}\right|-d(d+1) / 2=d\left|V^{\prime}\right|-$ $d(d+1) / 2$, implying that $V^{\prime *}=V^{\prime}$ and $G^{* *}$ is minimally rigid, and hence that $G^{\prime}$ is rigid and persistent, because of Lemma 2 and the fact that $G^{\prime *}$ is a subgraph of $G^{*}$, which is minimally rigid.

Let us now suppose to obtain a contradiction that $\left|E^{\prime *}\right|<d\left|V^{\prime}\right|-d(d+1) / 2$. Since $E^{*}=\left(E^{*} \cap E^{\prime}\right) \cup$ $\left(E^{*} \cap\left(E \backslash E^{\prime}\right)\right)=E^{\prime *} \cup\left(E^{*} \cap\left(E \backslash E^{\prime}\right)\right)$, this implies that $\left|E^{*}\right| \leqslant\left|E^{\prime *}\right|+\left|E^{*} \cap\left(E \backslash E^{\prime}\right)\right| \leqslant\left|E^{\prime *}\right|+\left|E \backslash E^{\prime}\right|<d\left|V^{\prime}\right|-$ $d(d+1) / 2+\left|E \backslash E^{\prime}\right|$. To compute $\left|E \backslash E^{\prime}\right|$, consider the closed subgraph $G^{\prime}=\left(V^{\prime}, E^{\prime}\right)$. Since $V^{\prime}$ has at least $d$ vertices, because of Theorem 14, it contains at least one vertex $i$ that does not belong to any closed subgraph of $G$ having less than $d-1$ vertices. Therefore, because of Theorem 14, there exists a path from this vertex $i$ to any vertex of $G$ with a positive DOF count. Since the subgraph $G^{\prime}$ containing $i$ is closed, this means that all the vertices of $G$ with a positive DOF count are also in $G^{\prime}$, or in other words, all the vertices of $V \backslash V^{\prime}$ have an out-degree $d$. Therefore and again using the fact that $G^{\prime}$ is closed, it follows that $\left|E \backslash E^{\prime}\right|=d\left|V \backslash V^{\prime}\right|$ and hence $\left|E^{*}\right|<d\left|V^{\prime}\right|-d(d+1) / 2+d\left|V \backslash V^{\prime}\right|=d|V|-d(d+1) / 2$, which contradicts the equality $\left|E^{*}\right|=d|V|-d(d+1) / 2$. Hence $\left|E^{\prime *}\right|$ cannot be less than $d\left|V^{\prime}\right|-d(d+1) / 2$ and the proof is complete for Case 1 .

- Case 2: $G=(V, E)$ is a persistent graph in $\Re^{d}$ with at least $d$ vertices such that at least one vertex $v \in V$ has an out-degree larger than $d$. Let $G^{\prime}=\left(V^{\prime}, E^{\prime}\right)$ be a closed subgraph of $G$ having more than $d-1$ vertices. From Theorem 9, we know that $G^{\prime}$ is persistent if and only if any subgraph $G^{\prime \prime}$ obtained by removing edges leaving vertices with out-degree larger than or equal to $d+1$ until all the vertices have out-degree smaller than or equal to $d$ are rigid. Let us consider such a $G^{\prime \prime}$, and build a graph $G^{*}$ by adding all the vertices of $V \backslash V^{\prime}$, and for each added vertex $j$ in $V \backslash V^{\prime}$, adding $\min \left\{d, d_{G}^{+}(j)\right\}$ of the outgoing edges from vertex $j$ appearing in $G$. One can see that $G^{*}$ can be obtained from $G$ by removing certain edges leaving vertices with $d^{+} \geqslant d+1$ until all vertices have $d^{+} \leqslant d$. Since $G$ is persistent, because of Proposition 6, we have that $G^{*}$ is persistent. One can also see that $G^{\prime \prime}$ is a closed subgraph of $G^{*}$ : if there was an edge leaving $G^{\prime \prime}$ and arriving in $G^{*} \backslash G^{\prime \prime}$, the same edge would leave $G^{\prime}$ and arrive in $G \backslash G^{\prime}$, which would contradict the hypothesis that $G^{\prime}$ is a closed subgraph. Since all the vertices of $G^{*}$ have $d^{+} \leqslant d$, we can apply the arguments in Case 1 to guarantee that $G^{\prime \prime}$ is persistent, and therefore rigid. This can be done for all $G^{\prime \prime}$, and $G^{\prime}$ is thus persistent.

In $\mathfrak{R}^{3}$, Proposition 16 leads to the following corollary, which indicates that all the closed subgraphs of a persistent graph except (possibly) a specific subgraph are persistent. The corollary also presents a necessary and sufficient condition for a graph to have all its closed subgraphs persistent.

Corollary 17. Let $G$ be a persistent graph in $\mathfrak{R}^{3}$ with at least three vertices. Then any closed subgraph $G^{\prime}$ of $G$ is persistent unless $G^{\prime}$ consists of two disconnected vertices. In other words, $G$ has a non-persistent closed subgraph if and only if it contains two vertices each having 3 DOFs.

\section{Structural persistence}

In this section, we focus on a subtle property of the persistent graphs. It gives rise to a problem in contemplating the task of maintaining the shape of a formation. This problem appears when any single agent can move to a position which satisfies all the constraints on it once all the other agents are fixed, but it is not possible to satisfy all the constraints on all the agents at the same time.

Consider a persistent graph $G=(V, E)$ in $\mathfrak{R}^{d}(d \in$ $\{2,3, \ldots\})$, and let $p$ be a representation with distance set $\bar{d}$. Consider nearby representations $p^{\prime}$, with $d\left(p, p^{\prime}\right)<\varepsilon$. In these nearby representations, there may exist a subset $\tilde{V} \subset V$ for which there are positions that are fitting for $\bar{d}$, whatever are the positions of the vertices of $\tilde{V} \subset V$, but for certain positions of the vertices in $\tilde{V}$, there exists no position assignment for the vertices in $V \backslash \tilde{V}$ such that each vertex in $V \backslash \tilde{V}$ is simultaneously fitting. For example, consider the three-dimensional persistent graph $\bar{G}$ shown in Fig. 4(a), an associated set $\bar{d}$ of desired lengths $d_{i j}>0$ for all the edges $\overrightarrow{(i, j)}$, and a realization $\bar{p}$ of $\bar{d}$ in agreement with Fig. 4(a). Identify $\tilde{V}$ with $\{1,2\}$. Since the vertices 1 and 2 have zero out-degrees, they are at fitting positions for any representation of the graph, whatever the positions of 3, 4, 5 are. However, there are representations of $\bar{G}$ arbitrarily close to $\bar{p}$ (namely, those where the distance between vertices 1 and 2 differs from the value in $\bar{p}$ ) where the vertices 3,4 , and 5 cannot be at fitting positions at the same time. From the perspective of formations, in the formation represented by $\bar{G}$, there exist two leaders, 1 and 2, which are allowed to move freely in $\mathfrak{R}^{3}$ without any constraint. This freedom, however, makes it impossible in some cases for the agents 3, 4, and 5 to simultaneously meet all the distance constraints on them, although $\bar{G}$ is persistent, according

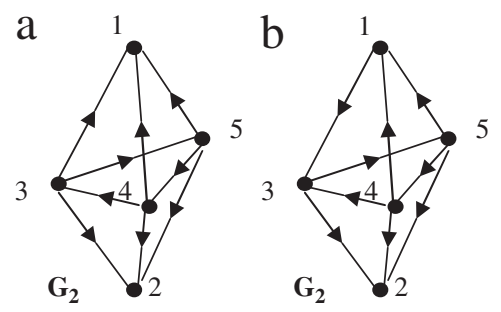

Fig. 4. (a) A persistent graph in $\mathfrak{R}^{3}$ which is not structurally persistent. (b) A structurally persistent graph in $\mathfrak{R}^{3}$. 

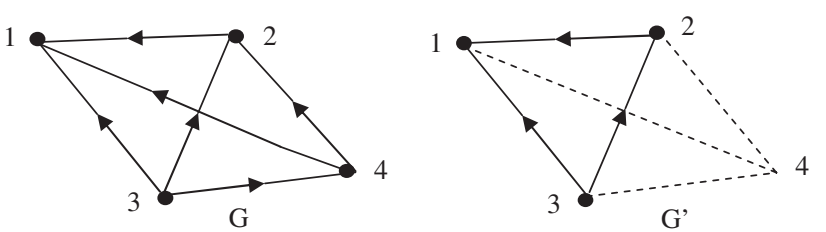

Fig. 5. A practically closed subgraph in $\mathfrak{R}^{2}$.

to the definition given in Section 2. In such a case, we will say that $\bar{G}$ fails to be structurally persistent. In comparison, the formation represented by Fig. 4(b) is structurally persistent, as shown in the sequel.

The existence of such persistent but not structurally persistent graphs in three- and higher dimensional spaces makes it necessary to analyse persistent graphs further and introduce the new concept of structural persistence that will be defined in this section. In $\mathfrak{R}^{2}$, however, there is no persistent graph that is not structurally persistent, as explained later in Theorem 21. To proceed with the analysis, let us consider a persistent graph $G=(V, E)$ in $\mathfrak{R}^{d}(d \in\{2,3, \ldots\})$ with a representation $p$. Let $\bar{d}$ be the set of distances corresponding to $p$. G fails to be structurally persistent if there exists a non-empty vertex subset $\tilde{V} \subset V$, a constant $\bar{\varepsilon}>0$ and a mapping $p_{\varepsilon}$ indexed by $\varepsilon$, $\left\{p_{\varepsilon}: \tilde{V} \rightarrow \mathfrak{R}^{d} \mid 0<\varepsilon \leqslant \bar{\varepsilon}\right\}$ such that for any $\varepsilon \leqslant \bar{\varepsilon}$ the following conditions hold:

(1) $d\left(p(i), p_{\varepsilon}(i)\right) \leqslant \varepsilon, \quad \forall i \in \tilde{V}$.

(2) For all $i \in \tilde{V}, p_{\varepsilon}(i)$ is a fitting position with respect to $\bar{d}$, irrespective of the positions of the vertices in $V \backslash \tilde{V}$.

(3) There exist no fitting representation $p^{\prime}: V \rightarrow \mathfrak{R}^{d}$ in $B(p, \varepsilon) \triangleq\left\{\bar{p}: V \rightarrow \mathfrak{R}^{d} \mid d(p, \bar{p}) \leqslant \varepsilon\right\}$, with respect to $\bar{d}$, such that $p^{\prime}(i)=p_{\varepsilon}(i), \quad \forall i \in \tilde{V}$.

If there exists no such subset for a persistent graph, then the graph is said to be structurally persistent. To analyse this concept further, we introduce a further notion: for a given directed graph $G=(V, E)$, a subgraph $G^{\prime}=\left(V^{\prime}, E^{\prime}\right)$ is a practically closed subgraph of $G$ if for any vertex $i \in V^{\prime}$, $d_{G^{\prime}}^{+}(i) \geqslant \min \left\{d, d_{G}^{+}(i)\right\}$, where $d_{G^{\prime}}^{+}(i)$ denotes the number of outgoing edges incident to the vertex $i$ of a graph $G^{\prime}$. We remark that a closed subgraph is always a practically closed subgraph, since each vertex of it satisfies the criterion defined above.

In the two-dimensional example shown in Fig. 5, where $V^{\prime}=$ $\{1,2,3\}, G^{\prime}$ is a practically closed subgraph of $G$ but not a closed subgraph of $G$. All the outgoing edges of 1 and 2 in $G$ remain in the subgraph $G^{\prime}$. Vertex 3, on the other hand, has two outgoing edges in $G^{\prime}$ (making $G^{\prime}$ a practically closed subgraph) and another one not in $G^{\prime}$. From a perspective of formations where vertices denote agents and edges denote awareness and distance constraints, in $G$, although 3 is aware of 4 , it may not be able to react to correctly maintain its distance from 4 because its position is locked by the constraints with respect to the vertices 1 and 2 .

The relation between structural persistence and practically closed subgraphs is examined in the following propositions.
Proposition 18. Consider a persistent graph $G=(V, E)$ in $\mathfrak{R}^{d}$ $(d \in\{2,3, \ldots\})$ with a representation $p$ and a set $\bar{d}$ of distances corresponding to $p$. Let $G^{\prime}=\left(V^{\prime}, E^{\prime}\right)$ be a subgraph of $G$ where $V^{\prime}$ is a non-empty vertex subset of $V$. Then, $G^{\prime}$ is a practically closed subgraph of $G$ if and only if there exist a constant $\varepsilon>0$ and a mapping $p_{\bar{\varepsilon}}$ indexed by $\bar{\varepsilon},\left\{p_{\bar{\varepsilon}}: V^{\prime} \rightarrow \mathfrak{R}^{d} \mid 0<\bar{\varepsilon} \leqslant \varepsilon\right\}$ such that for any $\bar{\varepsilon} \leqslant \varepsilon$ the following conditions hold:

(1) $d\left(p(i), p_{\bar{\varepsilon}}(i)\right) \leqslant \bar{\varepsilon}, \forall i \in V^{\prime}$, for all $p_{\bar{\varepsilon}}$ in the mapping set.

(2) For all $i \in V^{\prime}, p_{\bar{\varepsilon}}(i)$ is a fitting position with respect to $\bar{d}$, irrespective of the positions of the vertices in $V \backslash V^{\prime}$.

Proof. Suppose that $G^{\prime}$ is not a practically closed subgraph of $G$. Then, there exists a vertex $i_{0}$ in $V^{\prime}$ such that $d_{G^{\prime}}^{+}\left(i_{0}\right)<\min \left\{d, d_{G}^{+}\left(i_{0}\right)\right\}$, i.e., $i_{0}$ has less than $d$ neighbours in $V^{\prime}$ and a non-empty set of neighbours in $V \backslash V^{\prime}$. Consider a mapping $p^{\prime}: V^{\prime} \rightarrow \mathfrak{R}^{d}$. If we are allowed to change the position of a vertex $i$ in $V$ without modifying the positions of the others, we can generically (i.e., for almost all representations $p$ ) make up to $d$ edges leaving $i$ active (if the edges leaving $i$ are less than $d$ in number, all of them can be made active). The position $p^{\prime}\left(i_{0}\right)$ can thus be fitting only if it satisfies at least one distance constraint towards avertex in $V \backslash V^{\prime}$, which depends on the positions of the vertices in $V \backslash V^{\prime}$. Hence, it is not possible to find a mapping $p_{\bar{\varepsilon}}$ that satisfies (ii) for any $\bar{\varepsilon}>0$.

Conversely, let us suppose that we have a practically closed subgraph $G^{\prime} \subset G$. Let $p^{\prime}$ be a representation of $G^{\prime}$, fitting temporarily just for the distance set induced by the restriction of $p$ to $G^{\prime}$, and consider it as a part of a representation $\bar{p}$ (i.e., $p^{\prime}(i)=\bar{p}(i)$ for all $i \in V^{\prime}$ ) of $G$. Suppose further that $d(p, \bar{p})<\varepsilon$. It remains to show that $\bar{p}$ is fitting in $G$ (with respect to $\bar{d}$ ). Given any $i_{0} \in V^{\prime}$, if there exists no edge from $i_{0}$ to $V \backslash V^{\prime}$, then $\bar{p}\left(i_{0}\right)=p^{\prime}\left(i_{0}\right)$ is obviously fitting for any such $\bar{p}$. If there exists at least one edge from $i_{0}$ to $V \backslash V^{\prime}$, then, by definition of practically closed subgraph, there exist at least $d$ edges in $E^{\prime}$ leaving from $i_{0}$. Since the position of $i_{0}$ is fitting in $G^{\prime}$, at least $d$ of the edges $\overrightarrow{\left(i_{0}, j\right)} \in E^{\prime}$ are active. This means (generically) that there exists an $\varepsilon>0$ such that for $d(p, \bar{p}) \leqslant \varepsilon$ and $d\left(p, p^{\prime}\right) \leqslant \varepsilon$, there is only one position for $i_{0}$, i.e., $p^{\prime}\left(i_{0}\right)$ to satisfy all the constraints on $i_{0}$ following from the active edges in $G^{\prime}$. So considering $G$ and $\bar{p}$, there is no position other than $\bar{p}\left(i_{0}\right)$ that would satisfy all the constraints that $\bar{p}\left(i_{0}\right)$ already satisfies in $G^{\prime} . \bar{p}\left(i_{0}\right)$ is thus a fitting position, whatever the positions of the neighbours of $i_{0}$ in $V \backslash V^{\prime}$ are.

Proposition 19. Consider a persistent graph $G=(V, E)$ in $\mathfrak{R}^{d}$ $(d \in\{2,3, \ldots\})$ with a representation $p$ and a set $\bar{d}$ of distances corresponding to p. $G$ is structurally persistent if and only if every non-empty practically closed subgraph of $G$ is persistent.

Proof. Suppose that $G$ fails to be structurally persistent; then the three conditions of the definition of failure of structural persistence are satisfied for some $\tilde{V}=V^{\prime}$, say, $\varepsilon>0$ and a mapping set $\left\{p_{\bar{\varepsilon}}: V^{\prime} \rightarrow \mathfrak{R}^{d} \mid 0<\bar{\varepsilon} \leqslant \varepsilon\right\}$. By Proposition 18, the holding of the first two conditions implies that there is a nonempty practically closed subgraph $G^{\prime}=\left(V^{\prime}, E^{\prime}\right)$ of $G$. Now if $G^{\prime}$ is persistent, $p_{\bar{\varepsilon}}$ is congruent to the restriction $p_{V^{\prime}}$ of $p$ to $V^{\prime}$, 
i.e., $p_{\bar{\varepsilon}}$ can be obtained from $p_{V^{\prime}}$ applying a unique Euclidean transformation (rotation and translation). Applying the same transformation on the whole representation $p$ will then give a representation $p^{\prime}$ that is congruent to $p$ (and thus fitting for $\bar{d}$ ) and satisfying $p^{\prime}(i)=p_{\bar{\varepsilon}}(i)$ for all $i \in V^{\prime}$. This contradicts Condition 3 of the definition of failure of structural persistence. Hence if $G$ fails to be structurally persistent, it contains at least one non-empty practically closed subgraph which is not persistent.

Conversely, if there exists a practical closed subgraph $G^{\prime}$ that is not persistent then there exist a constant $\varepsilon>0$ and a mapping set $\left\{p_{\bar{\varepsilon}}: V^{\prime} \rightarrow \mathfrak{R}^{d} \mid 0<\bar{\varepsilon} \leqslant \varepsilon\right\}$ such that for any $0<\bar{\varepsilon} \leqslant \varepsilon, p_{\bar{\varepsilon}}$ is fitting (for the restriction of $\bar{d}$ to $E^{\prime}$ ) but not congruent to the restriction $p_{V^{\prime}}$ of $p$ to $V^{\prime}$. Note that $p_{\bar{\varepsilon}}$ satisfies Conditions 1 and 2 of the definition of structural persistence failure. Moreover, there exists no representation $p^{\prime}$ of $G$ fitting for $\bar{d}$ and at the same time satisfying $p^{\prime}(i)=p_{\bar{\varepsilon}}(i)$ for all $i \in V^{\prime}$. If it were the case, the persistence of $G$ implies that such a representation would be congruent to $p$, and thus that $p_{\bar{\varepsilon}}$ would be congruent to $p_{V^{\prime}}$, which contradicts the hypothesis. Hence Condition 3 for the failure of structural persistence is also satisfied implying that $G$ is not structurally persistent.

Proposition 19 states that a graph is not structurally persistent if and only if it contains a practically closed subgraph that is not persistent. Development of this notion leads to the following proposition, which gives another necessary and sufficient condition for a persistent graph to be structurally persistent.

Proposition 20. Let $G=(V, E)$ be a persistent graph in $\Re^{d}$. $G$ is structurally persistent if and only if every closed subgraph of $G$ with less than d vertices is persistent.

Proof. If there is a non-persistent closed subgraph $G^{\prime}$ of $G$ with less than $d$ vertices, then $G^{\prime}$ is also a non-persistent practically closed subgraph of $G$ which implies $G$ is not structurally persistent. Conversely, assume $G$ has no non-persistent closed subgraph with less than $d$ vertices. Consider an arbitrary practically closed subgraph $G^{\prime}=\left(V^{\prime}, E^{\prime}\right)$ of $G$, and the graph $G^{\prime \prime}$ obtained from $G$ by removing all the edges leaving $V^{\prime}$ and arriving at $V \backslash V^{\prime}$. By definition of a practically closed subgraph, any such edge $e$ has to leave a vertex in $V^{\prime}$ which has at least $d$ outgoing edges arriving at $V^{\prime}$. Therefore, by Proposition 6, removal of $e$ does not affect persistence. Thus the persistence of $G$ implies the persistence of $G^{\prime \prime}$. Observe also that no closed subgraph on less than $d$ vertices is affected or created by the removal of the edges $e$ leaving $V^{\prime}$ and arriving at $V \backslash V^{\prime}$, since such a subgraph contains only vertices with out-degree smaller than $d-1$. Since $G$ has no non-persistent closed subgraph with less than $d$ vertices, by Proposition 16, this implies that all the closed subgraphs of $G^{\prime \prime}$ are persistent. Since $G^{\prime}$ is by construction a closed subgraph of $G^{\prime \prime}$, this implies that $G^{\prime}$ is persistent. Hence, from Proposition 19, we have that $G$ is structurally persistent.

The following theorems, which are two main results of the section, and which immediately follow from Proposition 20, give more explicit necessary and sufficient condition for twoand three-dimensional persistent graphs to be structurally persistent. Theorem 22, moreover, gives more insight for the problem encountered in the example in Fig. 4.

Theorem 21. Any persistent graph $G \in \mathfrak{R}^{2}$ is structurally persistent.

Proof. By Proposition 20, a persistent graph in $\mathfrak{R}^{2}$ is structurally persistent if and only if it contains no non-persistent closed subgraph with less than two vertices, i.e., no nonpersistent closed subgraph with one vertex. The result follows then by the observation that a graph having only one vertex is always persistent.

Theorem 22. A persistent graph $G=(V, E)$ in $\mathfrak{R}^{3}$ is structurally persistent if and only if there is at most one leader in $G$.

Proof. From Proposition 20, we have that $G$ is structurally persistent if and only if there is no non-persistent closed subgraph of $G$ with less than three vertices. Observing that in $\mathfrak{R}^{3}$, the only possible non-persistent closed subgraph with less than three vertices is the edgeless subgraph composed of two vertices each having $3 \mathrm{DOFs}$, we see that for $G$, having no non-persistent closed subgraph with less than three vertices is equivalent to having at most one leader. Hence, the result immediately follows.

Next, we seek to provide a streamlined test for structural persistence. The test starts by looking at vertices with positive DOF. There may be another way of doing this. We shall adopt as a hypothesis that the graph of interest is known to be persistent.

Lemma 23. A graph $G=(V, E),|V| \leqslant d$ is rigid in d dimensions if and only if it is a complete graph, i.e., its undirected structure is $K_{|V|}$.

Proof. The condition is sufficient since a complete graph is trivially always rigid. Let us now prove that the condition is necessary. Consider a rigid realization on $|V| \leqslant d$ vertices. The positions of these vertices (almost always) define a $|V|$ dimensional subspace of $\Re^{d}$. Since the realization is rigid in $\Re^{d}$, it must also be rigid in this subspace. We must then have $|E| \geqslant|V||V|-|V|(|V|+1) / 2=|V|(|V|-1) / 2$, which is only possible if $G$ is the complete graph.

Corollary 24. Let $G$ be a persistent graph. A closed subgraph of $G$ on $d$ or fewer vertices is persistent if and only if it is a complete graph.

Proof. Observe that since there are at most $d$ vertices in the subgraph, all the out-degrees are smaller than $d$. The subgraph is thus always constraint consistent. By Lemma 23 , it is then rigid (and thus persistent) if and only if it is a complete graph. 


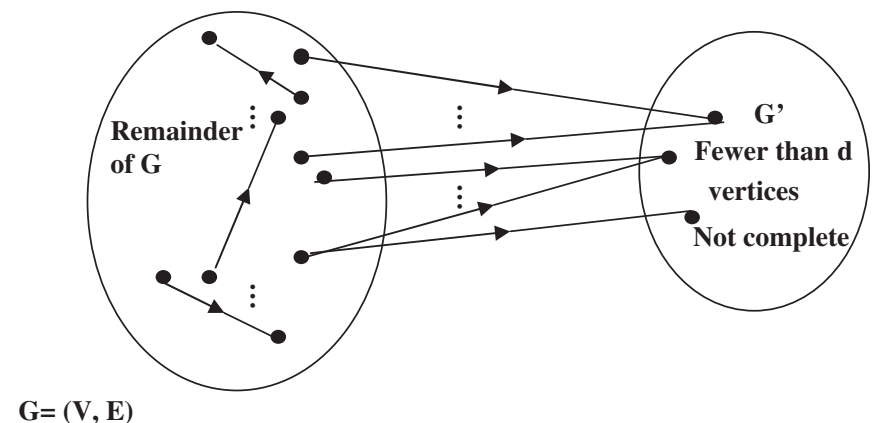

Fig. 6. General form of a persistent graph $G$ which is not structurally persistent in $\Re^{d} . G^{\prime}$ contains all the vertices that belong to at least one closed subgraph on less than $d$ vertices.

Theorem 25. Let $G=(V, E)$ be a persistent graph and $V_{c}$ be the set of vertices that belong to at least one closed subgraph on less than d vertices. $G$ is structurally persistent if and only if its restriction to $V_{c}$ is a complete graph.

Proof. Consider all the closed subgraphs on less than $d$ vertices. By definition of a closed subgraph and of $V_{c}$, their union $G_{c}$ is a closed subgraph with $V_{c}$ as vertex set. By Theorem 14, we know that $\left|V_{c}\right| \leqslant d-1 . G_{c}$ is thus a closed subgraph on less than $d$ vertices. Observe also that $G_{c}$ is the restriction of $G$ to $V_{c}$ (as is the case for any closed subgraph). We have thus to prove that $G$ is structurally persistent if and only if $G_{c}$ is a complete graph. Suppose first that $G_{c}$ is not a complete subgraph. By Corollary 24, it is then not persistent. Since there is in this case a non-persistent closed subgraph on less than $d$ vertices, $G$ is not structurally persistent. The condition is thus necessary. Conversely, as shown in Fig. 6, suppose that $G$ is not structurally persistent. There exists by Proposition 20 a non persistent closed subgraph $G^{\prime}=\left(V^{\prime}, E^{\prime}\right)$ on less than $d$ vertices. By Corollary 24, $G^{\prime}$ is thus not a complete graph. By definition of $V_{c}, V^{\prime}$ is a subset of $V_{c}$. Moreover, by the definition of a closed subgraph, if two vertices of $V^{\prime}$ are not connected in $G^{\prime}$, they are not connected in $G$ nor in $G_{c}$. So, if $G^{\prime}$ is a noncomplete graph, so is $G_{c}$. We have thus proved that $G$ is structurally persistent if and only if $G_{c}$ is a complete graph.

As depicted in Fig. 4(a), where one can verify that $V_{c}=\{1,2\}$ and $E_{c}$ is an empty edge set, this means the closed subgraph $G_{c}=\left(V_{c}, E_{c}\right)$ is not a complete graph and $\left|V_{c}\right|<3$, hence by Theorem 25 the corresponding $G$ is not structurally persistent. In contrast, $V_{c}$ is empty for the graph $G$ in $4(\mathrm{~b})$, hence $G$ is structurally persistent.

It is not difficult to conceive an algorithm for verifying/detecting $V_{c}$, and for checking its completeness. The algorithm is based on building the reachability graphs for those vertices with out-degree at most $d-2$ (Krumke $\&$ Noltemeier, 2005).

\section{Assurance of structural persistence in $\mathfrak{R}^{3}$}

In Section 5, we have seen that for a directed graph, persistence is not enough to ensure structural persistence, which

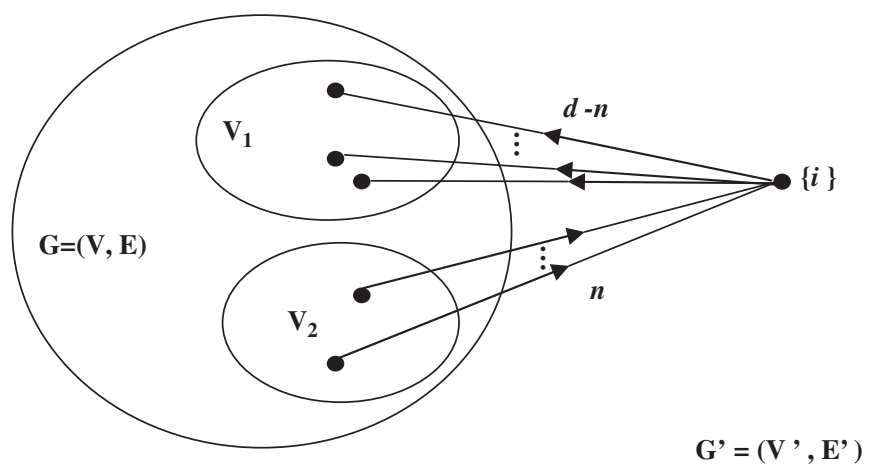

Fig. 7. A directed $d$-vertex addition.

is obviously desirable in three dimensional formations. The example depicted in Fig. 4 demonstrates that the way DOFs happen to be allocated to the vertices of a directed graph can determine structural persistence or otherwise; we will study this phenomena more systematically in this section. In an application scenario, this corresponds to giving/restricting the autonomy of certain agents (abstracted as DOF of vertices) of the formation (Yu, Fidan, \& Anderson, 2005).

We first study the properties of the directed version of Henneberg-like vertex addition (Eren et al., 2005; Hendrickx, Anderson et al., 2005) in three and higher dimensions, which is an abstraction of the event that new agents join a formation, one at a time. We give examples of applying such operations to manipulate DOF allocation of persistent graphs, in particular, in $\Re^{3}$.

Let us consider a persistent graph $G=(V, E)$ in $\mathfrak{R}^{d}(d \in$ $\{2,3, \ldots\})$ where $|V| \geqslant d$. A directed $d$-vertex addition, DVA $(d, n)$ as depicted in Fig. 7 where $n \in\{0, \ldots, d\}$, transforms $G$ to another persistent graph $G^{\prime}=\left(V^{\prime}, E^{\prime}\right)$ where $V^{\prime}=V \cup\{i\}$, $E^{\prime}=E \cup\left\{\overrightarrow{i, k)}: \forall k \in V_{1}\right\} \cup\left\{\overrightarrow{(j, i)}: \forall j \in V_{2}\right\}, V_{1}, V_{2} \subseteq$ $V, V_{1} \cap V_{2}=\emptyset,\left|V_{1}\right|=d-n,\left|V_{2}\right|=n$, and $D O F(j) \geqslant 1, \forall j \in$ $V_{2},{ }^{3}$ provided that the vertices of $V_{1} \cup V_{2}$ do not lie in any $q$-dimensional affine hyperplane where $q<d$.

We note that from Lemma 5, constraint consistence is preserved with the directed $d$-vertex addition defined above. Moreover, from the following lemma which is drawn from Whiteley (1996a, 1997), we see that the rigidity is also preserved.

Lemma 26 (Whiteley (1996a, 1997)). A graph obtained by adding one vertex to a graph $G=(V, E)$ in $\mathfrak{R}^{d}$ and d edges from this vertex to other vertices of $G$ is generically rigid if and only if $G$ is generically rigid.

Hence by Theorem 3, the graph obtained after applying a directed $d$-vertex addition on a persistent graph in $\Re^{d}$ is persistent, i.e., the $d$-directed vertex addition defined above preserves the persistence of the graphs.

\footnotetext{
${ }^{3}$ Non-existence of $V_{2}$ means the corresponding $\operatorname{DVA}(d, n)$ cannot be performed for the graph.
} 


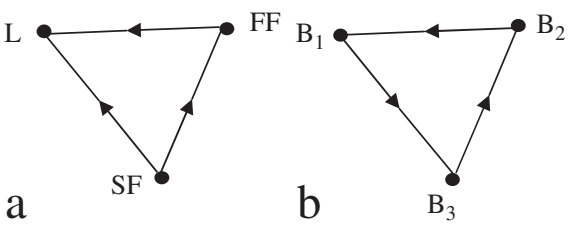

Fig. 8. The two directed triangular seeds.

Remark 27. Consider a persistent graph $G=(V, E)$ in $\mathfrak{R}^{d}$. Let $G^{\prime}=\left(V^{\prime}, E^{\prime}\right)$ be the graph obtained by applying the operation $D V A(d, n)$ to $G$, where $V^{\prime}=V \cup\{i\}$. Then we have:

(1) $D O F_{G^{\prime}}(i)=n$.

(2) $D O F_{G^{\prime}}(j) \leqslant D O F_{G}(j), \quad \forall j \in V$.

(3) $D O F_{G}(j)-D O F_{G^{\prime}}(j) \in\{0,1\}, \quad \forall j \in V$.

(4) $S(V, G)=S\left(V^{\prime}, G^{\prime}\right)$.

In the remaining part of this section, we only consider $\mathfrak{R}^{3}$, although results can be easily expanded to higher dimensions. As a more convenient nomenclature in $\mathfrak{R}^{3}$, we use the term directed trilateration operation, abbreviated $\mathrm{DT}(\cdot), \mathrm{DT}(n)$ in place of directed 3-vertex addition or $\operatorname{DVA}(3, n)$.

An undirected graph formed by a sequence of trilateration operations starting with an initial undirected triangle, often called a trilateration graph, is guaranteed to be generically rigid in $\mathfrak{R}^{3}$ and globally rigid in $\mathfrak{R}^{2}$ (Eren et al., 2004). Note that a seed with three vertices is needed to initiate a trilateration sequence. However, two different directed triangular seeds can start a directed trilateration operation in $\mathfrak{R}^{3}$ as defined in Fig. $8(\mathrm{a})$ and (b) are called the leader-first follower-second follower $(L-F F-S F)$ and the balanced triangle $\left(B_{1} B_{2} B_{3}\right.$ as three "co-leaders") seeds, respectively.

Remark 28. The leader-first follower-second follower seed is analogous to the leader-follower structure defined for a two-dimensional cycle-free graph (Hendrickx, Anderson et al., 2005). The set of DOF counts of the seed vertices is $\{3,2,1\}$. The balanced triangle is nothing more than a directed triangle (cycle) in a cyclic graph and the corresponding DOF count set is $\{2,2,2\}$.

Specifically in the application to three-dimensional agent formations, note the meanings of the DT $(i)$ operation for different $i$ can be interpreted as follows:

- DT(3) means election of a new leader.

- DT(2) may result in either breaking/restoring the balanced control structure, or election of a new first-follower.

- DT(1) may also result in either breaking/restoring the balanced control structure at more a detailed level, or creation/change of second follower.

- DT(0) preserves the control structure and no decision has to be made by pre-existing agents.

Noting that in a three-dimensional persistent graph, there are at most six DOFs (as opposed to at most three DOFs in the

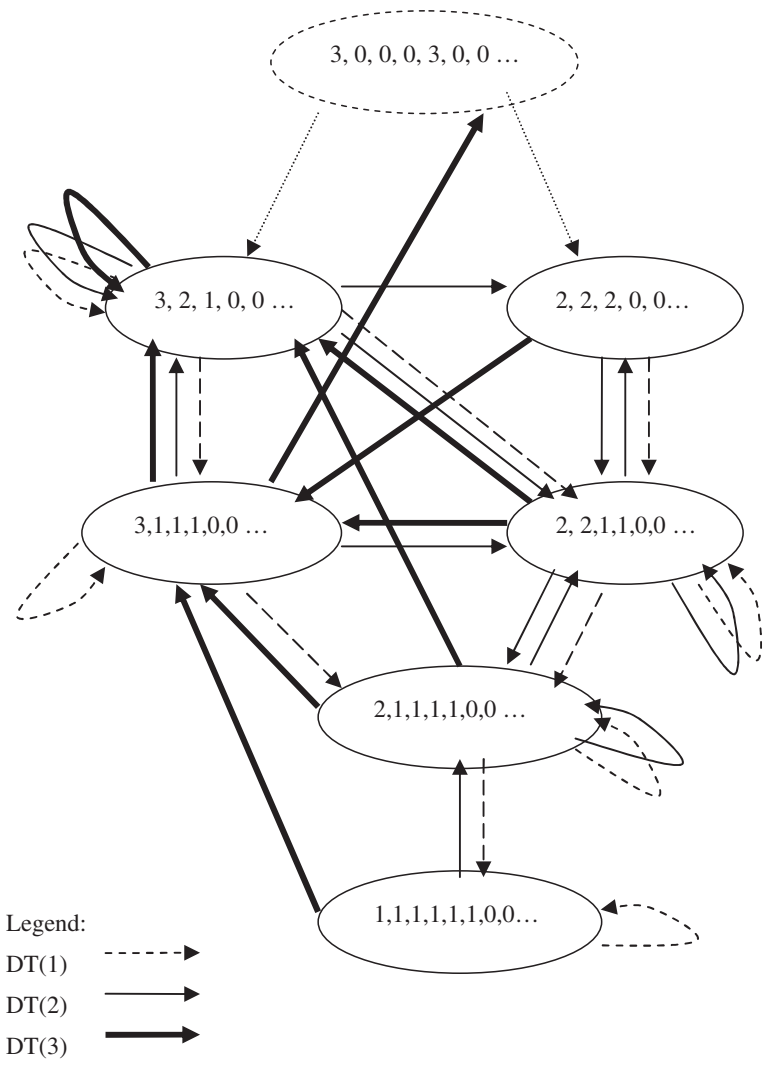

Fig. 9. The state transition diagram for directed trilaterations.

$\mathfrak{R}^{2}$ case) to be allocated among the vertices, we can list the following six types of DOF allocation which we call $D O F$ allocation states $S_{1}-S_{6}$ defined in terms of DOF counts of vertices as follows:

$S_{1}=\{3,2,1,0,0, \ldots\}, \quad S_{2}=\{2,2,2,0,0, \ldots\}$,

$S_{3}=\{3,1,1,1,0,0, \ldots\}, \quad S_{4}=\{2,2,1,1,0,0, \ldots\}$,

$S_{5}=\{2,1,1,1,1,0,0, \ldots\}, \quad S_{6}=\{1,1,1,1,1,1,0,0, \ldots\}$

Further, we define a transient type of DOF assignment $S_{0}=\{3,3,0,0, \ldots\}$, which can (only) be obtained by applying a DT(3) operation to $S_{3} . S_{0}$ is named "transient" because it apparently allows two leaders simultaneously in a formation, corresponding to failure of structural persistence and we want the DOF assignment to avoid this state. (An example of a graph that is in transient state $S_{0}$ can be seen in Fig. 4).

We study the transformational relationship between the possible distribution of DOFs by applying the appropriate DT(.) operation using the "state transition diagram" shown in Fig. 9. We have the following observations:

- Starting from any one of the two directed triangular seeds, we can build any graph with any of $S_{0}-S_{6}$ by adding at most three vertices using directed trilateration. 
- Any desired DOF reallocation pattern (with no allocation to a specific vertex) can be achieved by at most four directed trilaterations starting with any of the six types of DOF allocation.

- Any desired DOF reallocation pattern excluding $S_{0}$ can be achieved by at most three directed trilaterations starting with any other DOF allocation (excluding $S_{0}$ ).

The observed results above give an upper bound on the number of agents required in order to perform a system reconfiguration operation, such as replacement or elimination of leaders/firstfollower/second-follower, or change to balanced cooperative control by three "co-leaders". It also gives the possible consequences in a closing ranks problem ${ }^{4}$, where the lost agent has a certain positive number of DOFs .

Remark 29. A cycle-free graph in $\mathfrak{R}^{3}$ having more than 2 vertices is persistent if and only if it has a closed subgraph which is the leader-first follower-second follower triangle, and every other vertex has an out-degree larger than or equal to 3 (Yu et al., 2005). Refer to the state transition diagram in Fig. 9: the cycle-free and persistent properties require that the formation always has the DOF assignment of $3,2,1,0,0, \ldots$, i.e. stays in state $S_{1}$ in the process of building these cycle-free persistent graphs. This requirement eliminates the possibility of having a "transient" type DOF assignment, i.e., a state $S_{0}$, that creates a loss of structural persistence. Hence we can conclude that all cycle-free persistent graphs in $\mathfrak{R}^{3}$ are also structurally persistent.

\section{Concluding remarks}

In this paper, we have generalized the notion of persistence given in Hendrickx, Anderson et al. (2005) for two-dimensional directed graphs to dimensions higher than two, seeking to provide a theoretical framework for real world applications, which often are in three-dimensional space. We have verified that many of the properties already established for persistent graphs in $\mathfrak{R}^{2}$ are valid for higher dimensions as well. We have considered the directed paths in persistent graphs, derived some further properties of such graphs in three or higher dimensions, and given an easily checkable necessary condition (Proposition 16) for persistence.

Additionally, we have exposed and analysed a concept of structural persistence; failure of structural persistence arises roughly when any one vertex of a graph can satisfy all its constraints, but a subset of vertices cannot do so simultaneously. We have defined structurally persistent graphs, noting that for $d=2$, structural persistence is not different from persistence. We have studied the connection between the allocation of DOF across agents and the characteristics of persistence and/or structural persistence of a directed graph. We have proposed

\footnotetext{
4 The closing ranks problem for a given rigid formation which has lost a single agent, is to find new links between some agent pairs which when introduced cause the resulting formation to again be rigid.
}

directed $d$-vertex addition operations for $\mathfrak{R}^{d}$. We have also shown how to reallocate DOF among agents when the formation changes agent addition, to preserve persistence and/or structural persistence. Finally, we presented some results about cycle-free persistent graphs in $\mathfrak{R}^{3}$ which guarantee structural persistence.

The framework we presented in this paper leads to a new area of research and many open problems. For example, it appears to be important to seek systematic ways of constructing structurally persistent formations. It is essential to recast some of the existing problems posed using the notion of rigidity within the light of structural persistence. These problems include closing ranks, splitting and merging, formation manoeuver, etc. (Eren et al., 2004). Another potential focus is the real world implementation/deployment challenges for such formations. One challenge, among many other prominent problems, is to determine in what way we can ensure a level of robustness of sustaining structural persistence in the event of agent loss(es), link failure, or a combination of both. A closing ranks problem for a formation losing an agent now becomes one of restoring redundancy of structural persistence by adding further links. Yet another problem is to identify actual control laws which can be used to assure retention of structural persistence and inter-agent distances.

\section{Acknowledgements}

The work of Changbin Yu, Barış Fidan, and Brian D.O. Anderson is supported by National ICT Australia, which is funded by the Australian Government's Department of Communications, Information Technology and the Arts and the Australian Research Council through the Backing Australia's Ability Initiative. Changbin Yu was an Australia-Asia Scholar supported by the Australian Government's Department of Education, Science and Training through Endeavours Programs.

The work of Julien M. Hendrickx and Vincent D. Blondel is supported by the Belgian Programme on Interuniversity Attraction Poles initiated by the Belgian Federal Science Policy Office, and by the Concerted Research Action (ARC) "Large Graphs and Networks" of the French Community of Belgium. The scientific responsibility rests with its authors. Julien M. Hendrickx is a FNRS (Belgian Fund for Scientific Research) fellow.

\section{References}

Baillieul, J., \& Suri, A. (2003). Information patterns and hedging Brockett's theorem in controlling vehicle formations. Proceedings of the 42nd IEEE conference on decision and control (Vol. 1, pp. 556-563), December 2003.

Ceccarelli, N., Di Marco, M., Garulli, A., \& Giannitrapani, A. (2005). Collective circular motion of multi-vehicle systems with sensory limitations. Proceedings of the 44th IEEE conference on decision and control, and European control conference. (pp.740-745), Spain, December 2005.

Das, A., Fierro, R., Kumar, V., \& Ostrowski, J. P. (2002). A visionbased formation control framework. IEEE Transactions on Robotics and Automation, 18(5), 813-825.

Eren, T., Anderson, B.D.O., Morse, A.S., Whiteley, W., \& Belhumeur, P.N. (2004). Operations on rigid formations of autonomous agents. Communications in Information and Systems, 223-258. 
Eren, T., Whiteley, W., Anderson, B.D.O., Morse, A.S., \& Belhumeur, P.N. (2005). Information structures to secure control of rigid formations with leader-follower structure. Proceedings of the American control conference (pp. 2966-2971) Portland, June 2005.

Eren, T., Whiteley, W., Morse, A.S., Belhumeur, P.N., \& Anderson, B.D.O. (2003). Sensor and network topologies of formations with direction, bearing and angle information between agents. Proceedings of the 42nd IEEE Conference on decision and control (pp. 3064-3069) December 2003.

Gazi, V., \& Passino, K. M. (2003). Stability analysis of swarms. IEEE Transactions on Automatic Control, 48(4), 692-697.

Hendrickx, J.M., Anderson, B.D.O., Delvenne, J.-C., \& Blondel, V.D. (2005). Directed graphs for the analysis of rigidity and persistence in autonomous agent systems. International Journal of Robust Nonlinear Control, to available online, please refer to the link for correct reference information: http://www3.interscience.wiley.com/cgi-bin/abstract/113453596/ ABSTRACT

Hendrickx, J.M., Fidan, B., Yu, C., Anderson, B.D.O., \& Blondel, V.D. (2005). Rigidity and persistence of three and higher dimensional directed formations. Proceedings of the first international workshop on multi-agent robotic systems (pp. 39-46), Spain, September 2005.

Hubbard, S., Babak, B., Sigurdsson, S. T., \& Magnusson, K. G. (2004). A model of the formation of fish schools and migrations of fish. Ecological Modelling, 174, 359-374.

Ihle, I.-A.F., Jouffroy, J., \& Fossen, T.I., (2005). Formation control of marine surface craft using lagrange multipliers. Proceedings of the 44th IEEE conference on decision and control, and European control conference (pp. 752-758), Spain, December 2005.

Jadbabaie, A., Lin, J., \& Morse, A. S. (2003). Coordination of groups of mobile autonomous agents using nearest neighbor rules. IEEE Transactions on Automatic Control, 48(6), 988-1001.

Janson, S., Middendorf, M., \& Beekman, M. (2005). Honey bee swarms: How do scouts guide a swarm of uninformed bees?. Animal Behaviour, 70(1), 349-358.

Krumke, S.O., \& Noltemeier, H. (2005). Graphentheoretische Konzepte und Algorithmen. B.G. Teubner Verlag.

Lin, Z., Francis, B. A., \& Maggiore, M. (2005). Necessary and sufficient graphical conditions for formation control of unicycles. IEEE Transactions on Automatic Control, 50(1), 121-127.

Marshall, J. A., Broucke, M. E., \& Francis, B. A. (1963). Formations of vehicles in cyclic pursuit. IEEE Transactions on Automatic Control,49(11),

Olfati-Saber, R., \& Murray, R.M. (2002). Graph rigidity and distributed formation stablization of multi-agent systems. Proceedings of the 41 st IEEE conference on decision and control, (pp. 2965-2971).

Olfati-Saber, R., \& Murray, R. M. (2004). Consensus problems in networks of agents with switching topology and time-delays. IEEE Transactions on Automatic Control, 49(9), 1520-1533.

Ren, W., \& Beard, R. W. (2004). A decentralized scheme for spacecraft formation flying via the virtual structure approach. AIAA Journal of Gudiance, Control and Dynamics, 27(1), 73-82.

Tanner, H. G., Pappas, G. J., \& Kumar, V. (2004). Leader-to-formation stability. IEEE Transactions on Robotics and Automation, 20(3), 443-455.

Tay, T., \& Whiteley, W. (1985). Generating isostatic frameworks. Structural Topology, 11, 21-69.

Whiteley, W. (1996a). Matroid Theory, Contemporary Mathematics, (Vol. 197, pp. 171-311). Providence, RI: American Mathematical Society.

Whiteley, W. (1996b). Matroids from discrete geometry. In Bonin, J.E., Oxley, J.G., Servatius, B. (Eds.), Matroid Theory (Vol. 197, pp. 171-313).

Whiteley, W. (1997). Rigidity and scene analysis, Handbook of discrete and computational geometry, (pp. 893-916) Boca Raton, FL: CRC Press.

Yu, C., Fidan, B., \& Anderson, B.D.O. (2005). Persistence acquisition and maintenance for autonomous formations. Proceedings of the second international conference on intelligent sensors, sensor networks and information processing (pp. 379-384) Melbourne, Australia, December 2005.

Yu, C., Hendrickx, J.M., Fidan, B., \& Anderson, B.D.O. (2005). Structural persistence of three-dimensional autonomous formations. Proceedings of the first international workshop on multi-agent robotic systems (pp. 47-55) Barcelona, Spain, September 2005.

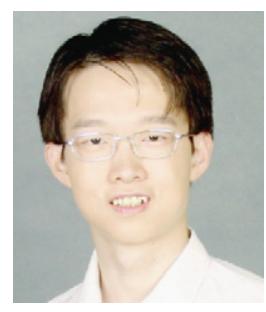

Changbin(Brad) Yu was born in Shandong, China. He received the B.Eng degree with first class honors in computer engineering from Nanyang Technological University (NTU), Singapore in 2004. Since 2005 he has been a $\mathrm{PhD}$ candidate under the supervision of Prof. Brian D.O. Anderson at the Australian National University (ANU), Canberra, Australia, and affliated also with the National ICT Australia. His research interests include autonomous formations, multi-agents systems, sensor networks and graph theory. He was a receipient of the Australian Government's Endeavour Asia Award and the international undergraduate scholarship award from the Republic of Singapore.

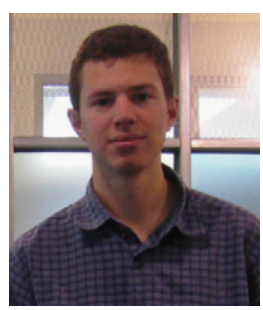

Julien Hendrickx is a Ph.D. candidate at the Université catholique de Louvain (Belgium) under the supervision of Prof. Vincent D. Blondel, and holds a FNRS (Belgian National Fund for Scientific Research) research appointment. $\mathrm{He}$ obtained an engineering degree in applied mathematics with highest honours from the same university in 2004.

Julien Hendrickx has been a visiting researcher at the Massachusetts Institute of Technology in 2006, at the National ICT Australia in 2005

and 2006, and at the University of Illinois at Urbana Champaign in 2003-2004. His main current research interests include multi-agents systems, swarming processes and graph theory.

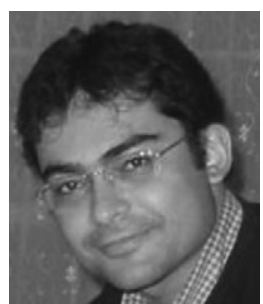

Barış Fidan received the B.S. degrees in electrical engineering and mathematics from Middle East Technical University, Turkey in 1996, the M.S. degree in electrical engineering from Bilkent University, Turkey in 1998, and the $\mathrm{Ph} . \mathrm{D}$. degree in Electrical Engineering-Systems at the University of Southern California, Los Angeles, USA in 2003. After working as a postdoctoral research fellow at the University of Southern California for one year, he joined the Systems Engineering and Complex Systems Program of National ICT Australia and the Research School of Information Sciences and Engineering of the Australian National University, Canberra, Australia in 2005, where he is currently a researcher. His research interests include autonomous formations, sensor networks, adaptive and nonlinear control, switching and hybrid systems, mechatronics, and various control applications including high performance and hypersonic flight control, semiconductor manufacturing process control, and disk-drive servo systems.

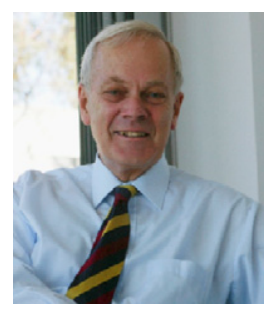

Professor Brian Anderson was born in Sydney, Australia. He took his undergraduate degrees in Mathematics and Electrical Engineering at Sydney University, and his doctoral degree in Electrical Engineering at Stanford University in 1966. His research interests have been principally in control systems, with significant work also in signal processing, telecommunications and circuit theory.

From 1990 to 1993 he was President of the International Federation of Automatic Control, and from 1994 to 2002 he was Director of the Research School of Information Sciences and Engineering at the Australian National University, and he served as President of the Australian Academy of Science from 1998 to 2002. He is currently Chief Scientist of National ICT Australia and Distinguished Professor in the Research School of Information Sciences and Engineering at ANU.

Professor Anderson is a Fellow of the Australian Academy of Science and Academy of Technology Sciences and Engineering, the Institute of Electrical and Electronic Engineers, the Royal Society, London; an Honorary Fellow of the Institution of Engineers, Australia and a Foreign Associate of the US National Academy of Engineering. He holds Honorary Doctorates from the Catholic University of Louvain, Belgium, the Swiss Federal Institute of Technology, and a number of Australian Universities. 


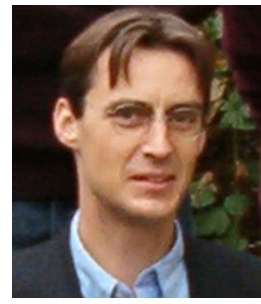

Vincent D. Blondel is Professor of Applied Mathematics and head of the Department of Mathematical Engineering at the Université catholique de Louvain (Belgium). He has held research and visiting appointments at Oxford University, at the Royal Institute of Technology in Stockholm, at INRIA France, at the Ecole Normale Supérieure in Lyon and at the University of Paris VII. He was visiting professor and Fulbright scholar at the Massachusetts Institute of Technology in 2005-2006. Dr. Blondel's major current research interests lie in several area of mathematical control theory and theoretical computer science. He has been a recipient of a Grant from the Trustees of the Mathematics Institute of Oxford University, the Prize Agathon De Potter of the Belgian Royal Academy of Science, the Prize Paul Dubois of the Montefiore Institute and the SIAM prize on control and systems theory. 